Minimal coupled cell networks

This article has been downloaded from IOPscience. Please scroll down to see the full text article. 2007 Nonlinearity 20193

(http://iopscience.iop.org/0951-7715/20/1/012)

View the table of contents for this issue, or go to the journal homepage for more

Download details:

IP Address: 193.136.31.121

The article was downloaded on 18/10/2012 at 10:08

Please note that terms and conditions apply. 


\title{
Minimal coupled cell networks
}

\author{
Manuela A D Aguiar ${ }^{1,2}$ and Ana Paula S Dias ${ }^{1,3}$ \\ ${ }^{1}$ Centro de Matemática da Universidade do Porto*, Rua do Campo Alegre, 687, 4169-007 Porto, \\ Portugal \\ ${ }^{2}$ Faculdade de Economia, Universidade do Porto, Rua Dr Roberto Frias, 4200-464 Porto, \\ Portugal \\ ${ }^{3}$ Dep. de Matemática Pura, Faculdade de Ciências, Universidade do Porto, Rua do Campo \\ Alegre, 687, 4169-007 Porto, Portugal \\ E-mail: maguiar@fep.up.pt and apdias@fc.up.pt
}

Received 6 April 2006, in final form 21 November 2006

Published 11 December 2006

Online at stacks.iop.org/Non/20/193

Recommended by Y G Kevrekidis

\begin{abstract}
It is known that non-isomorphic coupled cell networks can have equivalent dynamics. Such networks are said to be ODE-equivalent and are related by a linear algebra condition involving their graph adjacency matrices. A network in an ODE-equivalence class is said to be minimal if it has a minimum number of edges. When studying a given network in an ODE-class it can be of great value to study instead a minimal network in that class. Here we characterize the minimal networks of an ODE-equivalence class-the canonical normal forms of the ODE-class. Moreover, we present an algorithm that computes the canonical normal forms for a given ODE-class. This goes through the calculation of vectors with minimum length contained in a cone of a lattice described in terms of the adjacency matrices of any network in the ODE-class.
\end{abstract}

Mathematics Subject Classification: 34C15, 05C99, 15A36

\section{Introduction}

Many important real-world networks can be modelled by dynamical systems on a graph and therefore coupled cell networks. For example, many biological systems can be modelled by networks of nonlinear dynamical systems. See, for example, Stewart [8] and references therein. The nodes of the graph represent the cells and the edges represent the couplings, that is, the interactions between the cells (see figures $1-4$ ).

* CMUP is supported by FCT through POCTI and POSI of Quadro Comunitário de Apoio III (2000-2006) with FEDER and national fundings. 


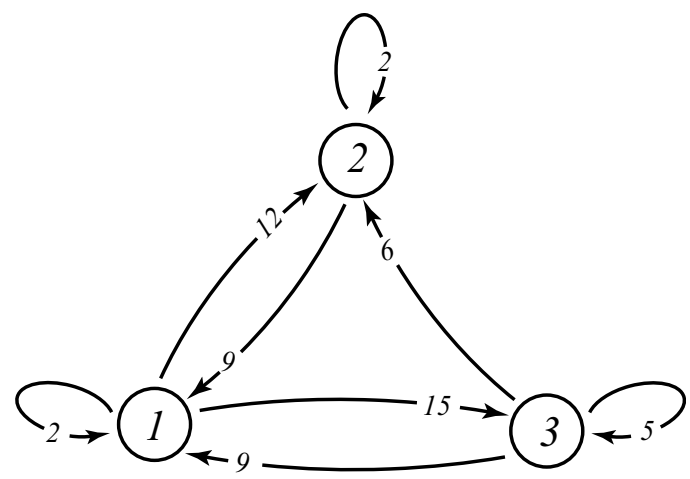

Figure 1. Coupled cell network $G_{1}$ with one cell-type and one edge-type.

Following Stewart et al [9] and Golubitsky et al [7], we associate to each coupled cell network a class of ordinary differential equations (ODEs) compatible with the structure of the network - the class of coupled cell systems. A vector field corresponding to the coupled cell system is called admissible and respects the topology of the network. For a survey of this formalism see Golubitsky and Stewart [6]. See also an alternative approach of Field [5].

It is possible for two non-isomorphic coupled cell networks to generate the same space of admissible vector fields, that is to be ODE-equivalent, see [7]. Thus the corresponding coupled cell systems have equivalent dynamics.

Dias and Stewart [4] prove that two coupled cell networks are ODE-equivalent if and only if they determine the same space of linear admissible vector fields-they are linearly equivalent.

As every coupled cell network in a given ODE-equivalence class determines the same dynamical behaviour we look for the set of coupled cell networks in the ODE-class that are more amenable to treat. We aim to find a kind of canonical normal forms - a set of networks such that the number of edges is minimal among all the networks of the ODE-equivalence class, which we call the minimal subclass.

Using the results of Dias and Stewart [4] on ODE and linear equivalence of networks, this problem can be posed in terms of the networks adjacency matrices. That is, the problem of finding all the minimal networks in a given ODE-equivalence class corresponds to find all the minimal bases (with non-negative integer entries) of the real vector space generated by the adjacency matrices of the networks in the ODE-class. The minimal bases define the adjacency matrices of the minimal networks.

For the coupled cell network represented in figure 1 it follows, from theorem 9.3 (see section 9), that the coupled cell network in figure 2 is the unique minimal network in its ODE-equivalence class.

The problem of finding the minimal subclass of an ODE-class of networks with more than one edge-type gets much more complicated and, in general, there is more than one minimal network. For example, using algorithm 7.1 based on theorem 6.7 , the minimal subclass of the coupled cell network in figure 3 is given by the minimal networks represented in figure 4 . Here, cells representing identical dynamical systems appear with the same symbol in the graph and identical edges symbolize identical couplings between the cells. In this case, the network has more than one edge-type and the cells are all isomorphic. Two cells are isomorphic if there is a bijection between the two sets of edges directed to them that preserves the edge-type. An adjacency matrix of a network with isomorphic cells is a $\mathbb{R}$-linear combination of the adjacency matrices of any network in its ODE-class. A graph where all cells are isomorphic is said to 


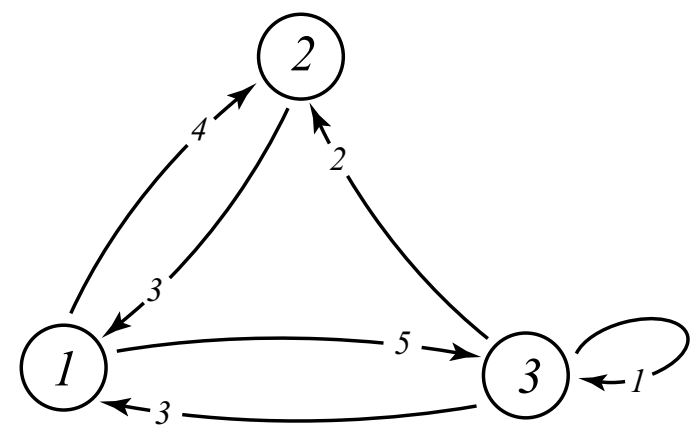

Figure 2. Minimal network $G_{2}$ of the ODE-equivalence class of the coupled cell network in figure 1.

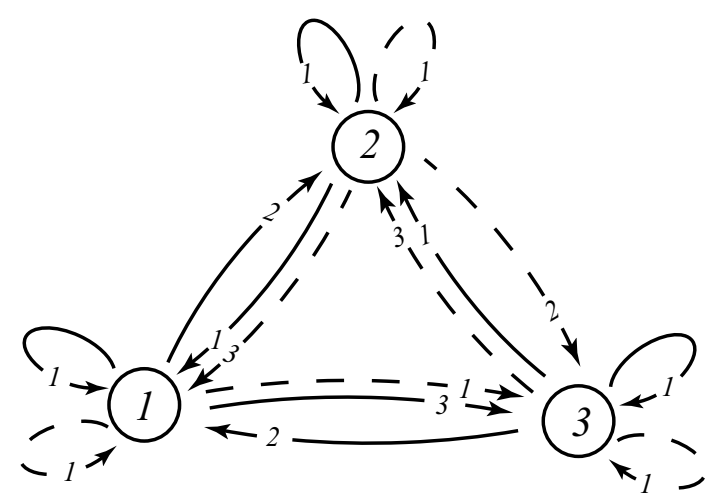

Figure 3. Coupled cell network with more than one edge type.

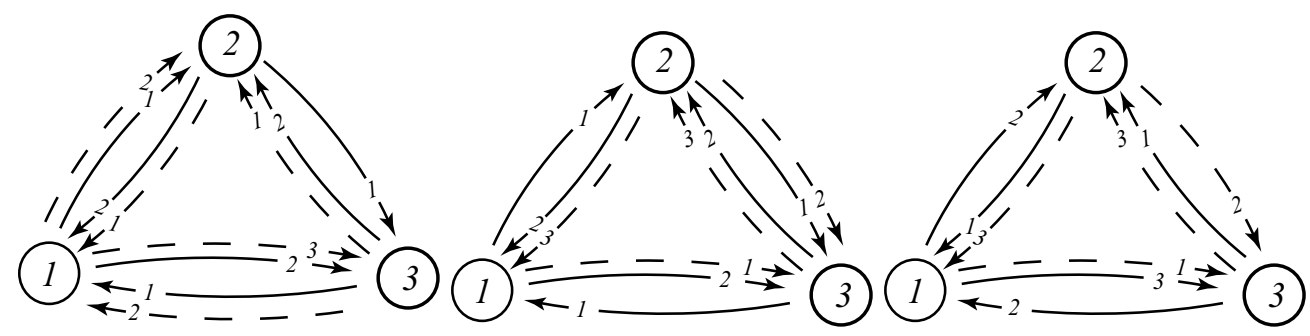

Figure 4. Minimal networks of the ODE-equivalence class of the coupled cell network of figure 3.

be homogeneous. Given an homogeneous graph in an ODE-class, we consider the lattice consisting of the vectors with integer entries in the real subspace generated by the adjacency matrices of the graph. The minimal networks are obtained by finding vectors (adjacency matrices) with shortest length in a cone of that lattice (whose rank grows with the number of edge-types).

In this paper, we describe the minimal subclass of a given coupled cell network and present an algorithm that computes it. The minimality of coupled cell networks basically reduces to the minimality of homogeneous networks, i.e. networks whose cells are all isomorphic. We address the case of homogeneous networks in theorem 6.7 and algorithm 7.1. 
The rest of the paper is organized in the following way. Section 2 reviews the basic concepts on coupled cell networks and coupled cell systems. Section 3 relates ODE and linear equivalence of networks. The definition of minimality of networks appears in section 4 . The minimality of homogeneous networks is addressed in section 5 and answered in section 6 . An algorithm is presented in section 7 and illustrated in section 8. The special case of identicaledge homogeneous networks is treated in section 9 . Finally, in section 10 we solve the general case using the results of section 6 .

\section{Coupled cell networks and systems}

In this section we review a few definitions and results on the theory of coupled cell networks introduced by Stewart et al [9] and Golubitsky et al [7]. A coupled cell network can be represented schematically by a directed graph (see, e.g. Tutte [10]) whose nodes correspond to cells and whose edges represent couplings. We employ the definition which permits multiple arrows and self-coupling [7].

Definition 2.1 ([7]). A coupled cell network $G$ consists of:

(a) A finite set $\mathcal{C}=\{1, \cdots, n\}$ of nodes or cells.

(b) An equivalence relation $\sim_{C}$ on cells in $\mathcal{C}$. The type or cell label of cell $c$ is the $\sim_{C^{-}}$ equivalence class $[c]_{C}$ of $c$.

(c) A finite set $\mathcal{E}$ of edges or arrows.

(d) An equivalence relation $\sim_{E}$ on edges in $\mathcal{E}$. The type or coupling label of edge $e$ is the $\sim_{E}$-equivalence class $[e]_{E}$ of $e$.

(e) Two maps $\mathcal{H}: \mathcal{E} \rightarrow \mathcal{C}$ and $\mathcal{T}: \mathcal{E} \rightarrow \mathcal{C}$. For $e \in \mathcal{E}$ we call $\mathcal{H}(e)$ the head of $e$ and $\mathcal{T}(e)$ the tail of $e$.

(f) Equivalent edges have equivalent tails and heads. That is, if $e_{1}, e_{2} \in \mathcal{E}$ and $e_{1} \sim_{E} e_{2}$, then

$$
\mathcal{H}\left(e_{1}\right) \sim_{C} \mathcal{H}\left(e_{2}\right) \quad \mathcal{T}\left(e_{1}\right) \sim_{C} \mathcal{T}\left(e_{2}\right) .
$$

We write $G=\left(\mathcal{C}, \mathcal{E}, \sim_{C}, \sim_{E}\right)$.

In a graphical representation of a coupled cell network, identical cells and identical edges are represented, respectively, by the same symbol. Multiple couplings of the same type between two cells are represented by just one arrow with the number of couplings attached to it. Figures 1-4 show examples of coupled cell networks of three identical cells, and so with one $\sim_{C}$-equivalence class, having self-coupling and multiarrows. The networks of figures 1 and 2 have only one type of edge, and so one $\sim_{E}$-equivalence class, whereas the networks in figures 3 and 4 have two types of edges, and so two $\sim_{E}$-equivalence classes.

Let $G=\left(\mathcal{C}, \mathcal{E}, \sim_{C}, \sim_{E}\right)$ be a coupled cell network as in definition 2.1. To each cell $c$, we can associate the set of edges directed to $c$, called the input set of $c$ and denoted by $I(c)$. An element of $I(c)$ is called an input edge of $c$. Two cells $c$ and $d$ in a network are said to be isomorphic if there is an edge-type preserving isomorphism, $\beta: I(c) \rightarrow I(d)$, between their input sets. Any such bijection $\beta$ is called an input isomorphism from cell $c$ to cell $d$. Denote the set of all those isomorphisms between any two isomorphic cells in the network, $\dot{\bigcup}_{c, d \in \mathcal{C}} B(c, d)$, where $\dot{U}$ indicates disjoint union, by $\mathcal{B}_{G}$. Observe that a natural product operation can be defined on $\mathcal{B}_{G}$ in the following way: $\beta_{2} \in B(c, d)$ can be multiplied by $\beta_{1} \in B(a, b)$ only when $b=c$, and in this case $\beta_{2} \beta_{1} \in B(a, d)$ is the usual composition of functions. Because the product of two elements in $\mathcal{B}_{G}$ is not always defined, it follows that $\mathcal{B}_{G}$ is a groupoid, which is called the symmetry groupoid of the network, and in general it is not a group. The structure of a network can thus be described in terms of its symmetry groupoid. 
Note that for any $c \in \mathcal{C}$, the subset $B(c, c)$ is always non-empty. Moreover, it is a group which is called the vertex group corresponding to $c$.

We make now precise the connection between coupled cell systems and coupled cell networks. To each coupled cell $c$ we associate a choice of cell phase space $P_{c}$ which we assume is a finite-dimensional real vector space. If cells $c$ and $d$ are in the same $\sim_{C}$-equivalence class, we require that $P_{c}=P_{d}$ and we identify the two spaces canonically. The total phase space $P$ of the coupled cell system is the direct product of the cell phase spaces, $\prod_{c \in \mathcal{C}} P_{c}$, and we employ the coordinate system $x=\left(x_{c}\right)_{c \in \mathcal{C}}$ on $P$.

Given a network $G$ and a fixed choice of the total phase space $P$, we describe now the coupled cell systems that correspond to the class of ODEs $\dot{X}=F(X), X \in P$ compatible with the structure of the network. The vector fields $F$ described in terms of the symmetry groupoid of $G$ are called admissible.

Let $\mathcal{D}=\left(d_{1}, \ldots, d_{s}\right)$ be any finite ordered subset of $s$ cells in $\mathcal{C}$ where the same cell can appear more than once in $\mathcal{D}$. Define $P_{\mathcal{D}}=P_{d_{1}} \times \cdots \times P_{d_{s}}$ and write $x_{\mathcal{D}}=\left(x_{d_{1}}, \ldots, x_{d_{s}}\right)$ where $x_{d_{j}} \in P_{d_{j}}$.

Given $c \in \mathcal{C}$, denote by $\mathcal{T}(I(c))$ the ordered set of cells $\left(\mathcal{T}\left(i_{1}\right), \ldots, \mathcal{T}\left(i_{s}\right)\right)$ where the arrows $i_{k}$ run through $I(c)$.

Suppose that $c, d$ are isomorphic and let $\beta$ be an input isomorphism between $I(c)$ and $I(d)$. Then for all $i \in I(c)$ we have $i \sim_{E} \beta(i)$, and so $\mathcal{T}(i) \sim_{C} \mathcal{T}(\beta(i))$. Consider now the ordered sets $\mathcal{D}_{1}=\mathcal{T}(I(c))=\left(\mathcal{T}\left(i_{1}\right), \ldots, \mathcal{T}\left(i_{s}\right)\right)$ and $\mathcal{D}_{2}=\mathcal{T}(I(d))=\left(\mathcal{T}\left(\beta\left(i_{1}\right)\right), \ldots, \mathcal{T}\left(\beta\left(i_{s}\right)\right)\right)$ of $\mathcal{C}$. We can define the pullback map

$$
\beta^{*}: P_{\mathcal{D}_{2}} \rightarrow P_{\mathcal{D}_{1}}
$$

by

$$
\left(\beta^{*}(z)\right)_{\mathcal{T}(j)}=z_{\mathcal{T}(\beta(j))}
$$

for all $\mathcal{T}(j) \in \mathcal{D}_{1}$ and $z \in P_{\mathcal{D}_{2}}$. Thus $x_{\mathcal{T}(I(c))}=\left(x_{\mathcal{T}\left(i_{1}\right)}, \ldots, x_{\mathcal{T}\left(i_{s}\right)}\right)$ and $\beta^{*}\left(x_{\mathcal{T}(I(d))}\right)=$ $\left(x_{\mathcal{T}\left(\beta\left(i_{1}\right)\right)}, \ldots, x_{\mathcal{T}\left(\beta\left(i_{s}\right)\right)}\right)$.

For a given cell $c$ the internal phase space is $P_{c}$ and the coupling phase space is $P_{\mathcal{T}(I(c))}=P_{\mathcal{T}\left(i_{1}\right)} \times \cdots \times P_{\mathcal{T}\left(i_{s}\right)}$ where as before $\mathcal{T}(I(c))$ denotes the ordered set of cells $\left(\mathcal{T}\left(i_{1}\right), \cdots, \mathcal{T}\left(i_{s}\right)\right)$.

Definition 2.2 ([7]). A vector field $f: P \rightarrow P$ is $\mathcal{B}_{G}$-equivariant or $G$-admissible if:

(a) For all $c \in \mathcal{C}$ the component $f_{c}(x)$ depends only on the internal phase space variables $x_{c}$ and the coupling phase space variables $x_{\mathcal{T}(I(c))}$; that is, there exists a smooth function $\hat{f}_{c}: P_{c} \times P_{\mathcal{T}(I(c))} \rightarrow P_{c}$ such that

$$
f_{c}(x)=\hat{f}_{c}\left(x_{c}, x_{\mathcal{T}(I(c))}\right) .
$$

(b) For all $c, d \in \mathcal{C}$ and $\beta \in B(c, d)$

$$
\hat{f}_{d}\left(x_{d}, x_{\mathcal{T}(I(d))}\right)=\hat{f}_{c}\left(x_{d}, \beta^{*}\left(x_{\mathcal{T}(I(d))}\right)\right)
$$

for all $x \in P$.

Define the relation $\sim_{I}$ of input-equivalence on $\mathcal{C}$ by: $c \sim_{I} d$ if and only if $c$ and $d$ are isomorphic cells. Observe that $\sim_{I}$ is an equivalence relation.

Theorem 2.3. Let $G=\left(\mathcal{C}, \mathcal{E}, \sim_{C}, \sim_{E}\right)$ be a coupled cell network and $\mathcal{B}_{G}$ the corresponding symmetry groupoid. A vector field $f: P \rightarrow P$ for a given choice of the $P_{c}$ is $\mathcal{B}_{G}$-equivariant 
if and only if for each $\sim_{I}$-equivalence class $\mathcal{Q}$ of $\mathcal{C}$, given (any) $c \in \mathcal{Q}$ :

(a) $\hat{f}_{c}$ is $B(c, c)$-invariant.

(b) For $d \in \mathcal{Q}$ such that $d \neq c$, given (any) $\beta \in B(c, d)$, we have

$$
\hat{f}_{d}\left(x_{d}, x_{\mathcal{T}(I(d))}\right)=\hat{f}_{c}\left(x_{d}, \beta^{*}\left(x_{\mathcal{T}(I(d))}\right)\right) .
$$

Proof. This is a generalization of [9, lemma 4.5] and is proved the same way.

Definition 2.4. Let $G$ be a coupled cell network. For a given choice of the $P_{c}$, define $\mathcal{F}_{G}^{P}$ to consist of all smooth $G$-admissible vector fields on $P$ which is a vector space over $\mathbb{R}$. Let $\mathcal{P}_{G}^{P}$ be the subspace of $\mathcal{F}_{G}^{P}$ consisting of the $G$-admissible polynomial vector fields on $P$, and let $\mathcal{L}_{G}^{P}$ be the subspace of $\mathcal{P}_{G}^{P}$ consisting of the $G$-admissible linear vector fields on $P$.

Remark 2.5. Let $G$ be a coupled cell network and $P$ a given choice of the total phase space consistent with $G$. By theorem 2.3, every smooth equivariant vector field $f \in \mathcal{F}_{G}^{P}$ is determined uniquely by its components $f_{c}$ where $c$ runs through a set of representatives for the $\sim_{I^{-}}$ equivalence classes. The only constraints on $f_{c}$ are that it depends only on $x_{c}, x_{\mathcal{T}(I(c))}$ and is invariant under the vertex group $B(c, c)$. Thus every smooth equivariant vector field $f$ is determined uniquely by a finite set of $B(c, c)$-invariant functions, where $c$ runs through a set of representatives of the $\sim_{I}$-equivalence classes. Moreover, if $d \sim_{I} c$ then $f_{d}$ is related to $f_{c}$ by a pullback map $\beta^{*}$ for $\beta \in B(c, d)$. In particular, if there is only one $\sim_{I}$-equivalence class for $\mathcal{B}_{G}$ then each $G$-admissible vector field is uniquely determined by a single mapping $f_{c}$ at some node $c$, which has to be invariant under the vertex group $B(c, c)$.

Example 2.6. We return to the coupled cell network $G_{1}$ of figure 1. Since all cells $c \in \mathcal{C}_{1}$ are identical, they have the same cell phase space. Suppose that the cell phase space is $\mathbb{R}^{k}$. Then the total phase space for $G_{1}$ is $P=\mathbb{R}^{k} \times \mathbb{R}^{k} \times \mathbb{R}^{k}$. Moreover, since all cells are isomorphic and so input-equivalent, any $f=\left(f_{1}, f_{2}, f_{3}\right) \in \mathcal{F}_{G_{1}}^{P}$ is uniquely determined by one of the components, say $f_{1}$, which has to be $B(1,1)$-invariant, and then we obtain $f_{i}$, for $i=2,3$, using a bijection $\beta_{i} \in B(1, i)$. Throughout, denote by $\left(x_{i}^{(j)}\right)$ the vector $\left(x_{i}, \ldots, x_{i}\right)$ where the variable $x_{i}$ appears $j$ times. Set

$$
x_{\mathcal{T}(I(1))}=\left(x_{1}^{(2)}, x_{2}^{(9)}, x_{3}^{(9)}\right) .
$$

The $\mathcal{B}_{G_{1}}$-equivariance condition implies the $B(1,1)$-invariance of $\hat{f}_{1}: P_{1} \times P_{\mathcal{T}(I(1))} \rightarrow P_{1}$. Thus

$$
f_{1}(x)=\hat{f}_{1}\left(x_{1}, x_{1}^{(2)}, x_{2}^{(9)}, x_{3}^{(9)}\right)=\hat{f}_{1}\left(x_{1}, \beta^{*}\left(x_{1}^{(2)}, x_{2}^{(9)}, x_{3}^{(9)}\right)\right)
$$

for all $\beta \in B(1,1)$, and so $\hat{f}_{1}$ is symmetric in the set of the last 20 variables. We show that by putting a bar over that set:

$$
f_{1}(x)=\hat{f}_{1}\left(x_{1}, \overline{x_{1}^{(2)}, x_{2}^{(9)}, x_{3}^{(9)}}\right) .
$$

By theorem 2.3 any $\mathcal{B}_{G_{1}}$-equivariant vector field $f: P \rightarrow P$ takes the form

$$
f\left(x_{1}, x_{2}, x_{3}\right)=\left(\hat{f}_{1}\left(x_{1}, \overline{x_{1}^{(2)}, x_{2}^{(9)}, x_{3}^{(9)}}\right), \hat{f}_{1}\left(x_{2}, \overline{x_{1}^{(12)}, x_{2}^{(2)}, x_{3}^{(6)}}\right), \hat{f}_{1}\left(x_{3}, \overline{x_{1}^{(15)}, x_{3}^{(5)}}\right)\right) .
$$




\section{ODE-equivalence and linear equivalence}

As pointed by Golubitsky et al [7], in the class of coupled cell networks that permits selfcoupling and multiarrows, it is possible for two non-isomorphic coupled cell networks $G_{1}$ and $G_{2}$ to generate the same space of admissible vector fields. This comparison of admissible vector fields for two coupled cell networks involves identifying cells in the two networks, a step that it is formalized in general in terms of a bijection between the two sets of cells. We follow the definition of ODE-equivalent coupled cell networks given by Dias and Stewart [4]. In what follows, given a coupled cell network $G_{i}$ and a choice of total phase space $P_{i}$ for $G_{i}$, we denote by $P_{i, c}$ the cell phase space corresponding to cell $c$ of $\mathcal{C}_{i}$.

Definition 3.1 ([4]). Two coupled cell networks $G_{1}$ and $G_{2}$ are $\gamma$-ODE-equivalent if:

(1) There is a bijection $\gamma: \mathcal{C}_{1} \rightarrow \mathcal{C}_{2}$ that preserves cell-equivalence and input-equivalence, such that:

(2) If we choose cell phase spaces $P_{c} \neq 0$ for $G_{1}$, and define the corresponding choice of cell phase spaces for $G_{2}$ by

$$
P_{2, \gamma(c)}=P_{1, c}
$$

so that the corresponding total phase spaces are

$$
P_{1}=\prod_{c \in \mathcal{C}_{1}} P_{1, c} \quad P_{2}=\prod_{c \in \mathcal{C}_{1}} P_{2, \gamma(c)}
$$

then:

(3) The condition

$$
\mathcal{F}_{G_{1}}^{P_{1}}=\mathcal{F}_{G_{2}}^{P_{2}}
$$

is satisfied.

Two coupled cell networks $G_{1}$ and $G_{2}$ are $O D E$-equivalent if they are $\gamma$-ODE-equivalent for some bijection $\gamma$.

We define now the notion of 'linear equivalence' between two networks. In [4] it is shown that two coupled cell networks are ODE-equivalent if and only if they are linearly equivalent. Basically, the ODE-equivalence reduces to 'linear equivalence', where two networks (with suitably identified phase spaces) are linearly equivalent if they determine the same space of linear admissible vector fields. Moreover, when deciding linear equivalence, it can without loss of generality be assumed that each cell phase space is one-dimensional since if (3.1) holds for some choice of nonzero cell phase spaces $P_{c}$, then it holds for all such choices (that are consistent with the structure of $G$ ).

Definition 3.2. Two coupled cell networks $G_{1}$ and $G_{2}$ are $\gamma$-linearly equivalent if there is a bijection $\gamma: \mathcal{C}_{1} \rightarrow \mathcal{C}_{2}$ that preserves cell-equivalence and input-equivalence such that if item (2) of definition 3.1 is satisfied then:

(3) The condition

$$
\mathcal{L}_{G_{1}}^{P_{1}}=\mathcal{L}_{G_{2}}^{P_{2}}
$$

is satisfied.

Two coupled cell networks $G_{1}$ and $G_{2}$ are linearly equivalent if they are $\gamma$-linearly equivalent for some $\gamma$.

Remark 3.3. Note that this definition is independent of the dimensions of the $P_{c}$. Moreover, the cells of $G_{2}$ can be renumbered so that $\gamma$ is the identity. 
Throughout, we denote by $M_{n \times n}\left(\mathbb{Z}_{0}^{+}\right)$the set of the square matrices of order $n$ and nonnegative integer entries, and by $\mathrm{I}_{n}$ the identity matrix in this set.

Example 3.4. The coupled cell network $G_{2}$ of figure 2 is an example of a network linearly equivalent to the network $G_{1}$ of figure 1 . Both graphs have only one cell-equivalence class and one input-equivalence class. Trivially, the identity function on $\mathcal{C}_{1}=\{1,2,3\}$ preserves both cell-equivalence and input-equivalence. Moreover, if we take $P_{1}=P_{2}=\mathbb{R}^{3}$ then $\mathcal{L}_{G_{1}}^{P_{1}}=\mathcal{L}_{G_{2}}^{P_{2}}$ since

$$
\mathcal{L}_{G_{1}}^{P_{1}}=\mathbb{R}\left\{\operatorname{Id}_{\mathbb{R}^{3}}, h_{1}\right\}=\mathcal{L}_{G_{2}}^{P_{2}}=\mathbb{R}\left\{\operatorname{Id}_{\mathbb{R}^{3}}, h_{2}\right\},
$$

where $\mathbb{I}_{\mathbb{R}^{3}}$ denotes the identity function on $\mathbb{R}^{3}$,

$$
\begin{aligned}
& h_{1}\left(x_{1}, x_{2}, x_{3}\right)=\left(2 x_{1}+9 x_{2}+9 x_{3}, 12 x_{1}+2 x_{2}+6 x_{3}, 15 x_{1}+5 x_{3}\right) \\
& h_{2}\left(x_{1}, x_{2}, x_{3}\right)=\left(3 x_{2}+3 x_{3}, 4 x_{1}+2 x_{3}, 5 x_{1}+x_{3}\right)
\end{aligned}
$$

and $\left(x_{1}, x_{2}, x_{3}\right) \in \mathbb{R}^{3}$. Note that (3.3) is equivalent to saying

$$
\mathbb{R}\left\{\operatorname{Id}_{3},\left(\begin{array}{rrr}
2 & 9 & 9 \\
12 & 2 & 6 \\
15 & 0 & 5
\end{array}\right)\right\}=\mathbb{R}\left\{\operatorname{Id}_{3},\left(\begin{array}{lll}
0 & 3 & 3 \\
4 & 0 & 2 \\
5 & 0 & 1
\end{array}\right)\right\} .
$$

The next theorem reduces ODE-equivalence to linear equivalence, and says that the cell phase spaces may be assumed one-dimensional in that context.

Theorem 3.5 ([4]). Let $\gamma: \mathcal{C}_{1} \rightarrow \mathcal{C}_{2}$ be a bijection that preserves cell-equivalence and input-equivalence. Then the following conditions on two networks $G_{1}, G_{2}$ are equivalent:

(a) $G_{1}$ and $G_{2}$ are $\gamma$-ODE-equivalent.

(b) $G_{1}$ and $G_{2}$ are $\gamma$-linearly equivalent.

(c) With the identification $\gamma: \mathcal{C}_{1} \rightarrow \mathcal{C}_{2}$, the spaces $\mathcal{L}_{G_{1}}^{P}$ and $\mathcal{L}_{G_{2}}^{P}$ are equal when all cell phase spaces are taken to be $\mathbb{R}$.

Proof. See theorem 7.1 and corollary 7.9 of [4].

Example 3.6. We return to example 3.4. Recall (3.3). From the above theorem it follows that the two coupled cell networks of figures 1 and 2 are ODE-equivalent.

\section{Minimality}

In this section we compare ODE-equivalent networks in terms of the number of edges.

Definition 4.1. Let $G$ be an $n$-cell coupled cell network. We denote by $[G]$ the class of all coupled cell networks that are ODE-equivalent to $G$. By theorem 3.5 we have that $[G]$ coincides with the class of all coupled cell networks that are linearly equivalent to $G$.

Note that for a given $n$-cell network $G=\left(\mathcal{C}, \mathcal{E}, \sim_{C}, \sim_{E}\right)$, the set

$$
\left\{\left(\mathcal{C}_{1}, \mathcal{E}_{1}, \sim_{C_{1}}, \sim_{E_{1}}\right) \in[G]: \operatorname{card}\left(\mathcal{E}_{1}\right) \leqslant \operatorname{card}(\mathcal{E})\right\} \subseteq[G],
$$

where $\operatorname{card}(\mathcal{E})$ denotes the cardinality of the set of edges $\mathcal{E}$, is finite. In particular, it follows that the set

$$
\left\{\operatorname{card}\left(\mathcal{E}_{1}\right):\left(\mathcal{C}_{1}, \mathcal{E}_{1}, \sim_{C_{1}}, \sim_{E_{1}}\right) \in[G]\right\}
$$


has a minimum. We introduce now some notation:

Definition 4.2. Given an $n$-cell network $G$ and the corresponding ODE-class $[G]$, let

$$
m_{[G]}=\min \left\{\operatorname{card}\left(\mathcal{E}_{1}\right):\left(\mathcal{C}_{1}, \mathcal{E}_{1}, \sim_{C_{1}}, \sim_{E_{1}}\right) \in[G]\right\}
$$

and

$$
\operatorname{Min}_{[G]}=\left\{\left(\mathcal{C}_{1}, \mathcal{E}_{1}, \sim_{C_{1}}, \sim_{E_{1}}\right) \in[G]: \operatorname{card}\left(\mathcal{E}_{1}\right)=m_{[G]}\right\}
$$

We call $\mathcal{M i n}_{[G]}$ the minimal subclass of $[G]$.

The minimal subclass $\mathcal{M i n}_{[G]}$ of $[G]$ is thus the subclass of networks of $[G]$ such that the number of edges is minimal among all the networks of $[G]$ - the subclass of the canonical normal forms. In this paper, we describe the minimal subclass $\mathcal{M i n}_{[G]}$ of $[G]$, given an $n$-cell network $G$. More precisely, we give an algorithm that computes $\mathcal{M i n}_{[G]}$. We start by addressing the question for networks with only one $\sim_{I}$-equivalence class (sections $5-9$ ). It follows then as a corollary an algorithm that computes $\mathcal{M i n}_{[G]}$ for any ODE-class $[G]$ (section 10).

Example 4.3. Consider the graph $G_{1}$ of figure 1. For this example we have $m_{\left[G_{1}\right]}=18$ and $\mathcal{M i n}_{\left[G_{1}\right]}=\left\{G_{2}\right\}$, where $G_{2}$ is the coupled cell network of figure 2. See section 9 for details.

\section{Homogeneous networks}

In this section we address the minimality of networks in terms of the number of edges for ODE-classes associated with networks that have only one $\sim_{I}$-equivalence class.

Definition 5.1. An homogeneous network is a coupled cell network with only one inputequivalence class. An identical-edge homogeneous cell network is an homogeneous network in which all edges in $\mathcal{E}$ are equivalent. A nonidentical-edge homogeneous cell network is an homogeneous network with more than one edge-equivalence class.

Note that homogeneous networks have only one cell-equivalence class since these have only one $\sim_{I}$-equivalence class and by definition the equivalence relation $\sim_{I}$ refines $\sim_{C}$.

The graphs of figures 1 and 2 are examples of identical-edge homogeneous coupled cell networks. Figure 5 shows an example of a non-identical-edge homogeneous network with two edge-types.

Definition 5.2. Let $G=\left(\mathcal{C}, \mathcal{E}, \sim_{C}, \sim_{E}\right)$ be an homogeneous network with $n$ cells, say $\mathcal{C}=\{1, \ldots, n\}$, and $m$ edge-types, with $\left[e_{1}\right]_{E}, \ldots,\left[e_{m}\right]_{E}$, the $\sim_{E}$-equivalence classes. We define the adjacency matrix of $G$ with respect to $\left[e_{l}\right]_{E}$, for $l=1, \ldots, m$, to be the $n \times n$ matrix $M_{(G, l)}$ with rows and columns indexed by the cells of $G$ in the following way: the $(i, j)$-entry of $M_{(G, l)}$ corresponds to the number of edges of type $\left[e_{l}\right]_{E}$ from cell $j$ to cell $i$. Thus the sum of the $j$ th entries of row $i$ of the matrices $M_{(G, l)}$, for $l=1, \ldots, m$, gives the number of input edges of cell $i$ with tail cell $j$.

Example 5.3. The adjacency matrices of the coupled cell network $G_{1}$ of figure 5 with respect to the two edge-types are

$$
M_{\left(G_{1}, 1\right)}=\left(\begin{array}{rrr}
2 & 9 & 9 \\
12 & 2 & 6 \\
15 & 0 & 5
\end{array}\right) \quad \text { and } \quad M_{\left(G_{1}, 2\right)}=\left(\begin{array}{ccc}
10 & 25 & 10 \\
10 & 20 & 15 \\
20 & 10 & 15
\end{array}\right) .
$$




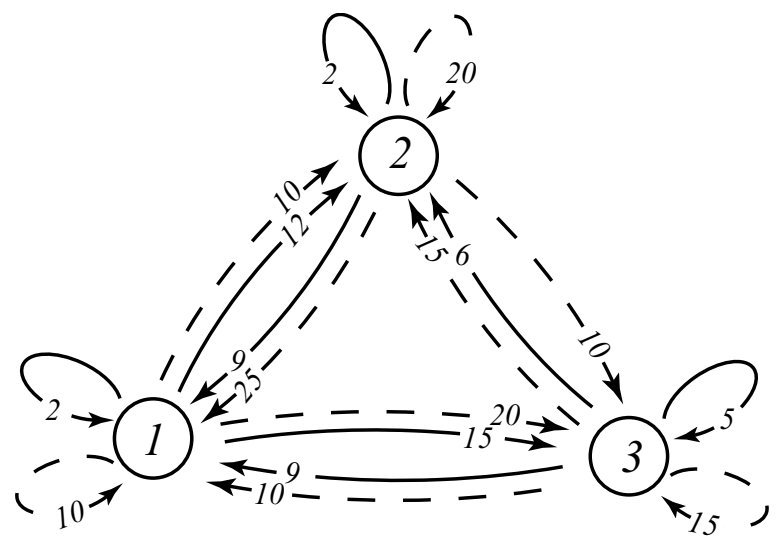

Figure 5. An example of a nonidentical-edge homogeneous network $G_{1}$.

\subsection{Linear equivalence of adjacency matrices}

We start by relating the adjacency matrices corresponding to linearly equivalent homogeneous networks. Definition 3.2 of linear equivalence is given by:

Proposition 5.4. Let $G_{1}$ be an homogeneous network with $n$ cells and $m_{1}$ edge-types, where $\mathcal{C}_{1}=\{1, \ldots, n\}$. Let $G_{2}$ be an homogeneous network with $n$ cells and $m_{2}$ edge-types. Denote by $M_{\left(G_{1}, l\right)}=\left[a_{i j}^{l}\right]_{1 \leqslant i, j \leqslant n}$ for $l=1, \ldots, m_{1}$, and $M_{\left(G_{2}, k\right)}=\left[b_{i j}^{k}\right]_{1 \leqslant i, j \leqslant n}$ for $k=1, \ldots, m_{2}$, the adjacency matrices of $G_{1}$ and $G_{2}$ with respect to $\left[e_{l}\right]_{E_{1}}$ and $\left[e_{k}\right]_{E_{2}}$, respectively. We have that $G_{1}$ and $G_{2}$ are linearly equivalent if and only if there is a bijection $\gamma: \mathcal{C}_{1} \rightarrow \mathcal{C}_{2}$ suc that:

$\mathbb{R}\left\{\mathrm{I}_{n},\left[a_{i j}^{1}\right]_{1 \leqslant i, j \leqslant n}, \cdots,\left[a_{i j}^{m_{1}}\right]_{1 \leqslant i, j \leqslant n}\right\}=\mathbb{R}\left\{\mathrm{I}_{n},\left[b_{\gamma(i) \gamma(j)}^{1}\right]_{1 \leqslant i, j \leqslant n}, \ldots,\left[b_{\gamma(i) \gamma(j)}^{m_{2}}\right]_{1 \leqslant i, j \leqslant n}\right\}$

Proof. Since both networks are homogeneous, trivially any bijection $\gamma: \mathcal{C}_{1} \rightarrow \mathcal{C}_{2}$ preserves cell-equivalence and input-equivalence. Thus definition 3.2 of linear equivalence translates to equation (5.5).

If necessary, we can relabel the cells of $G_{2}$ so that $\mathcal{C}_{1}=\mathcal{C}_{2}$ and $G_{2}$ is $\gamma$-linearly equivalent to $G_{1}$ where $\gamma$ is the identity on $\mathcal{C}_{1}=\{1, \ldots, n\}$. Moreover, since $G_{2}$ is linearly equivalent to $G_{1}$, then by proposition 5.4 it follows that

$\mathbb{R}\left\{\mathrm{I}_{n},\left[a_{i j}^{1}\right]_{1 \leqslant i, j \leqslant n}, \ldots,\left[a_{i j}^{m_{1}}\right]_{1 \leqslant i, j \leqslant n}\right\}=\mathbb{R}\left\{\mathrm{I}_{n},\left[b_{i j}^{1}\right]_{1 \leqslant i, j \leqslant n}, \ldots,\left[b_{i j}^{m_{2}}\right]_{1 \leqslant i, j \leqslant n}\right\}$.

Example 5.5. Using proposition 5.4, the network $G_{1}$ of figure 5 is linearly equivalent to the network $G_{2}$ of figure 6 , where $\gamma$ is the identity on $\{1,2,3\}$.

Remark 5.6. Let $G_{1}$ be an homogeneous network with $n$ cells. Let $M_{\left(G_{1}, 1\right)}, \ldots, M_{\left(G_{1}, m_{1}\right)}$ be the corresponding adjacency matrices. Then the set of matrices

$$
\mathcal{L}=M_{n \times n}(\mathbb{Z}) \cap \mathbb{R}\left\{\mathrm{I}_{n}, M_{\left(G_{1}, 1\right)}, \ldots, M_{\left(G_{1}, m_{1}\right)}\right\}
$$

with the usual sum of matrices is a lattice [1, chapter 3]: trivially, the set $\mathcal{L}$ is a discrete subgroup of $M_{n \times n}(\mathbb{R}) \cong \mathbb{R}^{n^{2}}$. 


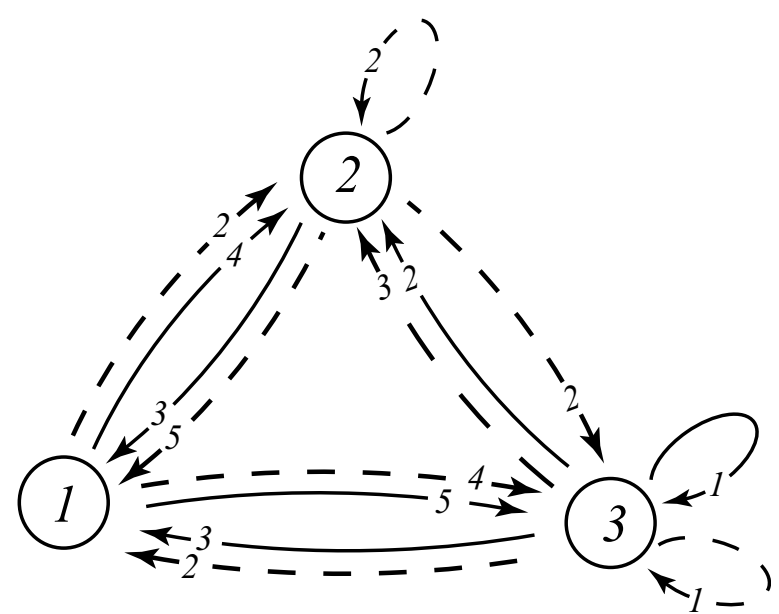

Figure 6. An example of a nonidentical-edge homogeneous network $G_{2}$ linearly equivalent to the graph of figure 5 .

The next lemma shows that the networks of the ODE-class $\left[G_{1}\right]$ have adjacency matrices lying in the cone $\mathcal{L} \cap M_{n \times n}\left(\mathbb{Z}_{0}^{+}\right)$.

Lemma 5.7. Let $G_{1}$ be an homogeneous network with $n$ cells, say $\mathcal{C}_{1}=\{1, \ldots, n\}$, and $m_{1}$ edge-types. Let $M_{\left(G_{1}, l\right)}=\left[a_{i j}^{l}\right]_{1 \leqslant i, j \leqslant n}$ for $l=1, \ldots, m_{1}$ be the corresponding adjacency matrices. Then $M=\left[m_{i j}\right]_{1 \leqslant i, j \leqslant n} \in M_{n \times n}\left(\mathbb{Z}_{0}^{+}\right)$is an adjacency matrix of a network $G$ (of $n$ cells) linearly equivalent to $G_{1}$ if and only if

$$
M \in \mathcal{L} \cap M_{n \times n}\left(\mathbb{Z}_{0}^{+}\right) .
$$

Proof. Note that each $M_{\left(G_{1}, l\right)}$ has the following properties:

$$
\begin{aligned}
& M_{\left(G_{1}, l\right)} \in M_{n \times n}\left(\mathbb{Z}_{0}^{+}\right) ; \\
& \sum_{j=1}^{n} a_{i j}^{l}=\sum_{j=1}^{n} a_{k j}^{l} \quad \text { for all } i, k .
\end{aligned}
$$

By proposition 5.4 we have that if $M=\left[m_{i j}\right]_{1 \leqslant i, j \leqslant n} \in M_{n \times n}\left(\mathbb{Z}_{0}^{+}\right)$is an adjacency matrix of a network $G$ (of $n$ cells) linearly equivalent to $G_{1}$ then condition (5.7) follows.

Suppose now that $M=\left[m_{i j}\right]_{1 \leqslant i, j \leqslant n} \in M_{n \times n}\left(\mathbb{Z}_{0}^{+}\right)$satisfies (5.7). In particular, since $G$ is homogeneous, it follows that

$$
\sum_{j=1}^{n} m_{i j}=\sum_{j=1}^{n} m_{k j} \quad \text { for all } i, k .
$$

\subsection{Minimality}

Given an homogeneous network $G$, we aim to describe the minimal subclass $\mathcal{M i n}_{[G]}$ of $[G]$. Recall definition 4.2. Using the above discussion on ODE-equivalence and linear equivalence, we can describe the graphs of $\mathcal{M i n}_{[G]}$ by computing the corresponding adjacency matrices. 
Moreover, we have:

Lemma 5.8. Let $G_{1}$ be an homogeneous network with $n$ cells. Let $\left[G_{1}\right]$ the corresponding ODE-class and $M_{\left(G_{1}, l\right)}$, for $l=1, \ldots, m_{1}$, the adjacency matrices of $G_{1}$. Let

$$
m=\operatorname{dim} \mathbb{R}\left\{\mathrm{I}_{n}, M_{\left(G_{1}, 1\right)}, \ldots, M_{\left(G_{1}, m_{1}\right)}\right\}-1 .
$$

Then the homogeneous networks of the minimal class $\mathcal{M i n}_{\left[G_{1}\right]}$ have m edge types.

Proof. Let $G_{2} \in \mathcal{M i n}_{\left[G_{1}\right]}$ and suppose that $G_{2}$ has $m_{2}$ edge types. Trivially, $m_{2} \geqslant m$ since

$$
\mathbb{R}\left\{\mathrm{I}_{n}, M_{\left(G_{1}, 1\right)}, \ldots, M_{\left(G_{1}, m_{1}\right)}\right\}=\mathbb{R}\left\{\mathrm{I}_{n}, M_{\left(G_{2}, 1\right)}, \ldots, M_{\left(G_{2}, m_{2}\right)}\right\}
$$

and so

$m_{2}+1 \geqslant \operatorname{dim} \mathbb{R}\left\{\mathrm{I}_{n}, M_{\left(G_{2}, 1\right)}, \ldots, M_{\left(G_{2}, m_{2}\right)}\right\}=\operatorname{dim} \mathbb{R}\left\{\mathrm{I}_{n}, M_{\left(G_{1}, 1\right)}, \ldots, M_{\left(G_{1}, m_{1}\right)}\right\}=m+1$.

If $m_{2}>m$ then

$$
\mathbb{R}\left\{\mathrm{I}_{n}, M_{\left(G_{2}, 1\right)}, \ldots, M_{\left(G_{2}, m_{2}\right)}\right\}=\mathbb{R}\left\{\mathrm{I}_{n}, M_{\left(G_{2}, i_{1}\right)}, \ldots, M_{\left(G_{2}, i_{k}\right)}\right\}
$$

for some set $\left\{i_{1}, \ldots, i_{k}\right\} \subset\left\{1, \ldots, m_{2}\right\}$ and $G_{2} \notin \operatorname{Min}_{\left[G_{1}\right]}$, a contradiction. Thus $m_{2}=m$.

Remark 5.9. Let $m$ be as defined in (5.10). Since $\operatorname{dim} \mathbb{R}\left\{\mathrm{I}_{n}, M_{(G, 1)}, \ldots, M_{\left(G, m_{1}\right)}\right\}$ equals $m+1$ we have that $\mathcal{L}$ is a lattice of rank $m+1$.

Definition 5.10. Given $M=\left[m_{i j}\right]_{1 \leqslant i, j \leqslant n} \in M_{n \times n}\left(\mathbb{Z}_{0}^{+}\right)$denote by

$$
l(M)=\sum_{i=1}^{n} \sum_{j=1}^{n} m_{i j} .
$$

More generally, for $u=\left(u_{1}, \ldots, u_{k}\right) \in\left(\mathbb{Z}_{0}^{+}\right)^{k}$ we call the length of $u$ the non-negative integer $l(u)$ defined by

$$
l(u)=\sum_{i=1}^{k} u_{i} .
$$

Note that if $w_{1}, \ldots, w_{m} \in\left(\mathbb{Z}_{0}^{+}\right)^{k}$ then

$$
l\left(\sum_{i=1}^{m} w_{i}\right)=\sum_{i=1}^{m} l\left(w_{i}\right) .
$$

Given a set of vectors $\left\{w_{1}, \ldots, w_{r}\right\} \subset\left(\mathbb{Z}_{0}^{+}\right)^{k}$ we denote

$$
l\left(\left\{w_{1}, \ldots, w_{r}\right\}\right)=\sum_{j=1}^{r} l\left(w_{j}\right) .
$$

We find the minimal subclass $\mathcal{M i n}_{[G]}$ in the following way:

Proposition 5.11. Let $G$ be an homogeneous network of $n$ cells and $[G]$ the corresponding ODE-class. Suppose that $M_{(G, 1)}, \ldots, M_{\left(G, m_{1}\right)}$ are the adjacency matrices of $G$. Let

$$
m=\operatorname{dim} \mathbb{R}\left\{\mathrm{I}_{n}, M_{(G, 1)}, \ldots, M_{\left(G, m_{1}\right)}\right\}-1 .
$$


An homogeneous network of the minimal class $\mathcal{M i n}_{[G]}$ has adjacency matrices $M_{1}, \ldots, M_{m}$ defined by:

(i) $\left\{\mathrm{I}_{n}, M_{1}, \ldots, M_{m}\right\} \subset M_{n \times n}\left(\mathbb{Z}_{0}^{+}\right)$;

(ii) $\left\{\mathrm{I}_{n}, M_{1}, \ldots, M_{m}\right\}$ is a basis of the real vector space $\mathbb{R} t\left\{\mathrm{I}_{n}, M_{(G, 1)}, \ldots, M_{\left(G, m_{1}\right)}\right\}$;

(iii) $\sum_{k=1}^{m} l\left(M_{k}\right)=m_{[G]}$.

Proof. By lemma 5.8 any graph $G_{2} \in \mathcal{M i n}_{[G]}$ has $m$ edge types. Item (i) follows by the definition of adjacency matrix of a network. Moreover, by lemma 5.7 and proposition 5.4 we have (ii). Condition (iii) follows from definition 4.2 of $\mathcal{M i n}_{[G]}$.

Remark 5.12. Note that if $M \in M_{n \times n}\left(\mathbb{Z}_{0}^{+}\right)$is an adjacency matrix of an homogeneous network, then $l(M) \geqslant n$ and so $l(M) \geqslant l\left(\mathrm{I}_{n}\right)$. Denote by $M_{0}=b_{0}=\mathrm{I}_{n}$. Assume the conditions of proposition 5.11 and denote $S=\mathbb{R}\left\{\mathrm{I}_{n}, M_{(G, 1)}, \ldots, M_{\left(G, m_{1}\right)}\right\}$. A set $\left\{M_{1}, \ldots, M_{m}\right\}$ satisfies (i)-(iii) if and only if it satisfies

(i) $\left\{M_{0}, M_{1}, \ldots, M_{m}\right\} \subset M_{n \times n}\left(\mathbb{Z}_{0}^{+}\right)$;

(ii) $\left\{M_{0}, M_{1}, \ldots, M_{m}\right\}$ is a basis of $S$;

(iii) $\sum_{k=0}^{m} l\left(M_{k}\right)=\min \left\{\sum_{k=0}^{m} l\left(b_{k}\right):\left\{b_{0}, b_{1}, \ldots, b_{m}\right\} \subset M_{n \times n}\left(\mathbb{Z}_{0}^{+}\right)\right.$is a basis of $\left.S\right\}$.

\section{Minimal bases}

By proposition 5.11 and remark 5.12 we have that the minimal subclass $\mathcal{M i n}_{[G]}$ of $[G]$ where $G$ is an homogeneous coupled cell network can be computed by describing the set of all possible adjacency matrices satisfying a minimality condition in terms of the length of those matrices. We abstract the problem in the following way.

Given a set of vectors $w_{1}, \ldots, w_{p} \in\left(\mathbb{Z}_{0}^{+}\right)^{k}$, denote by

$$
S=\mathbb{R}\left\{w_{1}, \ldots, w_{p}\right\}
$$

the real vector subspace of $\mathbb{R}^{k}$ generated by the vectors $w_{1}, \ldots, w_{p}$. Suppose that

$$
\operatorname{dim} S=r
$$

with $r \leqslant p$. We choose any $r$ linearly independent vectors from $w_{1}, \ldots, w_{p}$, say $w_{1}, \ldots, w_{r}$. Thus $w_{1}, \ldots, w_{r}$ form a basis of $S$. Denote by

$$
s=l\left(\left\{w_{1}, \ldots, w_{r}\right\}\right)
$$

Let

$$
\boldsymbol{B}=\left\{b: b=\left\{b_{1}, \ldots, b_{r}\right\} \subset\left(\mathbb{Z}_{0}^{+}\right)^{k} \text { is a basis of } S\right\} .
$$

Lemma 6.1. The set $\{l(b): b \in \boldsymbol{B}\}$ has a minimum.

Proof. As $\left\{w_{1}, \ldots, w_{r}\right\} \subset\left(\mathbb{Z}_{0}^{+}\right)^{k}$ is a basis of $S$ and $l\left(\left\{w_{1}, \ldots, w_{r}\right\}\right)=s$, it follows that the minimum of $\{l(b): b \in \boldsymbol{B}$ is at most $s$. Thus,

$$
r \leqslant \min \{l(b): b \in \boldsymbol{B}\} \leqslant s .
$$

Moreover,

$$
\min \{l(b): b \in \boldsymbol{B}\}=\min \{l(b): b \in \boldsymbol{B}, r \leqslant l(b) \leqslant s\},
$$

and $\{b \in \boldsymbol{B}: r \leqslant l(b) \leqslant s\}$ is a finite set. 
Definition 6.2. We call a basis $b \in \boldsymbol{B}$ of $S$ such that

$$
l(b)=\min \{l(\bar{b}): \bar{b} \in \boldsymbol{B}\}
$$

a minimal basis.

In this section we describe the set of all minimal bases $b \in \boldsymbol{B}$ of $S$. Moreover, we present an algorithm that computes this set.

\subsection{Sketch of the procedure}

The method we follow for finding the minimal bases of $S$ relies mostly on two steps:

(1) Find the set $C$ defined by

$$
C=\left\{w \in S \cap\left(\mathbb{Z}_{0}^{+}\right)^{k}: 0<l(w) \leqslant s-r+1\right\} .
$$

(2) Find all the minimal bases $b \in \boldsymbol{B}_{C}$ of $S$ where

$$
\boldsymbol{B}_{C}=\{b \in \boldsymbol{B}: b \subseteq C\} .
$$

\section{Remarks 6.3.}

(a) If $b=\left\{b_{1}, \ldots, b_{r}\right\} \subset\left(\mathbb{Z}_{0}^{+}\right)^{k}$ is a basis of $S$ and $r \leqslant l(b) \leqslant s$ then $b \subseteq C$. Moreover, $\boldsymbol{B}_{C} \subseteq \boldsymbol{B}$ and

$$
\min \{l(b): b \in \boldsymbol{B}\}=\min \left\{l(b): b \in \boldsymbol{B}_{C}\right\} .
$$

Thus the minimal bases $b=\left\{b_{1}, \ldots, b_{r}\right\}$ of $S$ are contained in $C$.

(b) Note that the set $C$ depends on the chosen basis $\left\{w_{1}, \ldots, w_{r}\right\}$ of $S$ which determines the length $s=l\left(\left\{w_{1}, \ldots, w_{r}\right\}\right)$. We show in theorem 6.7 that the minimal bases do not depend on the chosen set of the vectors $w_{i}$ that form a basis of $S$, nor on another choice of vectors (with non-negative integers coordinates) generating $S$.

\subsection{Finding $C$.}

Let $W$ be the $k \times r$ matrix with non-negative integer entries with columns given by the vectors $w_{1}, \ldots, w_{r}$ that we assume linearly independent and generating $S$. In notation

$$
W=\left(w_{1}|\cdots| w_{r}\right) \text {. }
$$

Denote by $M_{W}$ the set of all $r \times r$ matrices formed by $r$ rows of $W$ and with nonzero determinant and take

$$
\delta=\operatorname{gcd}\left\{\operatorname{det}(M): M \in M_{W}\right\} .
$$

Lemma 6.4. If $w \in S \cap\left(\mathbb{Z}_{0}^{+}\right)^{k}$ then there are rational numbers $x_{1}, \ldots, x_{r}$ whose denominators divide $\delta$ such that

$$
w=x_{1} w_{1}+\cdots+x_{r} w_{r} .
$$

Proof. By hypothesis $\left\{w_{1}, \ldots, w_{r}\right\}$ is a basis of $S$. Thus given $w \in S \cap\left(\mathbb{Z}_{0}^{+}\right)^{k}$, there are real numbers $x_{1}, \ldots, x_{r} \in \mathbb{R}$ such that $w=x_{1} w_{1}+\cdots+x_{r} w_{r}$. Moreover, we have that $w$ has integer components. Given $M \in M_{W}$, if we consider the linear system in the variables $x_{1}, \ldots, x_{r}$ obtained from $x_{1} w_{1}+\cdots+x_{r} w_{r}=w$ by keeping the rows corresponding to $M$, by Cramer's rule we have that the unique $x_{1}, \ldots, x_{r}$ are rational whose denominator divide the (nonzero) determinant of $M$. 
By the above lemma, we have that $C$ is the set of linear combinations of $w_{1}, \ldots, w_{r}$, say $x_{1} w_{1}+\cdots+x_{r} w_{r} \in\left(\mathbb{Z}_{0}^{+}\right)^{k}$, with rational coefficients $x_{1}, \ldots, x_{r}$ whose denominators divide $\delta$ and such that

$$
0<l\left(x_{1} w_{1}+\cdots+x_{r} w_{r}\right) \leqslant s-r+1 .
$$

Thus $x_{i}=p_{i} / q_{i}$ where $p_{i}, q_{i} \in \mathbb{Z}, q_{i} \neq 0$ and $\delta=n_{i} q_{i}$ for some $n_{i} \in \mathbb{Z}$. It follows then that

$$
\left(n_{1} p_{1}\right) w_{1}+\cdots+\left(n_{r} p_{r}\right) w_{r} \equiv 0 \quad(\bmod \delta) .
$$

We describe $C$ in the following way:

$$
C=\left\{\frac{1}{\delta} \sum_{j=1}^{r} y_{j} w_{j} \in\left(\mathbb{Z}_{0}^{+}\right)^{k}:\left\{\begin{array}{l}
\sum_{j=1}^{r} y_{j} w_{j} \equiv 0 \quad(\bmod \delta) \\
0<\frac{1}{\delta} l\left(\sum_{j=1}^{r} y_{j} w_{j}\right) \leqslant s-r+1
\end{array}\right\} .\right.
$$

\section{Remarks 6.5.}

(a) Note that $w_{1}, \ldots, w_{r} \in C$ since $\delta w_{i} \equiv 0 \quad(\bmod \delta)$ and for $i=1, \ldots, r$ we have $0<l\left(w_{i}\right) \leqslant s-r+1$

(b) The set $C$ is finite since it is a subset of the finite set formed by the vectors $w \in\left(\mathbb{Z}_{0}^{+}\right)^{k}$ such that $0<l(w) \leqslant s-r+1$.

\subsection{Finding the minimal bases}

In order to find the bases $b=\left\{b_{1}, \ldots, b_{r}\right\} \subseteq C$ of $S$ such that

$$
l(b)=\min \left\{l(b): b \in \boldsymbol{B}_{C}\right\}
$$

we partition the set $C$ by the length of its vectors:

$$
C=C_{1} \dot{\cup} C_{2} \dot{\cup} \ldots \dot{\cup} C_{t},
$$

where given $u, v \in C$

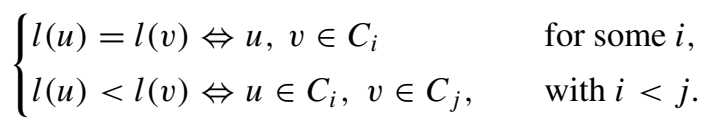

As $\left\{w_{1}, \ldots, w_{r}\right\}$ is a basis of $S$ and $w_{1}, \ldots, w_{r} \in C$, it follows that the real subspace of $\mathbb{R}^{k}$ generated by $C$ is $S$.

Definition 6.6. Let $S_{1}, S_{2}$ be real subspaces of $\mathbb{R}^{k}$. We denote

$$
S_{1}+S_{2}=\left\{u+v: u \in S_{1}, v \in S_{2}\right\} \text {. }
$$

Denote by $S_{i}, i=1, \ldots, t$, the subspace of $S$ generated by $C_{i}$. Thus

$$
S_{1}+\cdots+S_{t}=S \text {. }
$$

Moreover, there is $q$ such that $1 \leqslant q \leqslant t$ and

$$
\operatorname{dim}\left(S_{1}+\cdots+S_{q}\right)=r, \quad \operatorname{dim}\left(S_{1}+\cdots+S_{q-1}\right)<r .
$$

Thus we have

$$
S_{1}+\cdots+S_{q}=S
$$

We show below that the minimal bases of $S$ are contained in

$$
C_{1} \dot{\cup} \ldots \dot{\cup} C_{q} \text {. }
$$


Denote by $l_{i}$ the common length of any vector $u \in C_{i}$, for $i=1, \ldots, q$. Let

$$
\begin{aligned}
& d_{1}=\operatorname{dim} S_{1} \\
& d_{i}=\operatorname{dim}\left(\sum_{j=1}^{i} S_{j}\right)-\operatorname{dim}\left(\sum_{j=1}^{i-1} S_{j}\right), \quad 2 \leqslant i \leqslant q .
\end{aligned}
$$

Let $U_{1} \subseteq C_{1}$ be a set of $d_{1}$ linearly independent vectors. For $i=2, \ldots, q$, let $U_{i} \subseteq C_{i}$ be a set of $d_{i}$ vectors such that $U_{1} \cup \cdots \cup U_{i}$ is free.

Theorem 6.7. The minimum of the set

$$
\{l(b): b \in \boldsymbol{B}\}
$$

is

$$
m=\sum_{j=1}^{q} l_{j} d_{j} .
$$

The minimal bases $b \in \boldsymbol{B}$ of $S$ are the bases obtained by taking all the possible unions $U_{1} \cup \cdots \cup U_{q}$.

Proof. By lemma 6.1 the set $\{l(b): b \in \boldsymbol{B}\}$ has a minimum, say $m$.

Any set $U_{1} \cup \cdots \cup U_{q}=\left\{b_{1}, \ldots, b_{r}\right\}$ obtained as above is free and has $r$ vectors by construction. (Recall that by assumption $\operatorname{dim}\left(S_{1}+\cdots+S_{q}\right)=r$.) Thus it is a basis of $S$ and

$$
l\left(\left\{b_{1}, \ldots, b_{r}\right\}\right)=\sum_{j=1}^{q} l_{j} d_{j} .
$$

We prove now that the minimum $m$ of the set $\{l(b): b \in \boldsymbol{B}\}$ is

$$
\sum_{j=1}^{q} l_{j} d_{j}
$$

and that any minimal basis $b \in \boldsymbol{B}$ is the union $U_{1} \cup \cdots \cup U_{q}$ of sets $U_{i}$ constructed as above. In particular, it follows that $b \subseteq C$.

Starting with a different set of basis vectors $w_{i}$ of $S$, the set $C$ varies if the total length of the chosen basis, say $s^{*}$, differs from $s$. However, in this case, the new set, say $C^{*}$, would contain $C$ if $s^{*}>s$, and would be contained in $C$ if $s^{*}<s$. (Recall that $C^{*}$ would be formed by the non-null vectors in $S \cap\left(\mathbb{Z}_{0}^{+}\right)^{k}$ with length lower or equal to $s^{*}-r+1$.) In both cases, we have $\left(C_{1} \cup \cdots \cup C_{q}\right) \subseteq C^{*}$ since $\left(C_{1} \cup \cdots \cup C_{q}\right) \subseteq S$ and generates $S$. Note that in the second case, where $C^{*}$ would be contained in $C$, if $C_{1} \cup \cdots \cup C_{q} \nsubseteq C^{*}$, then we would have $C^{*}=C_{1} \cup \cdots \cup C_{s}$ where $s<q$ and so $S_{1}+\cdots+S_{s}=S$, which cannot happen by the definition of $q$ (recall (6.11)).

Suppose by contradiction that there is a basis $b=\left\{b_{1}, \ldots, b_{r}\right\} \in \boldsymbol{B}_{C}$ such that $b$ is not a union $U_{1} \cup \cdots \cup U_{q}$ of sets $U_{i}$ constructed as above and that

$$
l(b)=m \text {. }
$$

We have two cases to consider:

(i) $b \nsubseteq C_{1} \cup \cdots \cup C_{q}$. Then $b$ does not contain $r$ linearly independent vectors of $C_{1} \cup \ldots \cup C_{q}$. But $C_{1} \cup \ldots \cup C_{q}$ contains $r$ linearly independent vectors since by hypothesis $\operatorname{dim}\left(S_{1}+\cdots+S_{q}\right)=r$. Thus we can choose a vector $u \in C_{1} \cup \cdots \cup C_{q}$ such that $u \notin b$ and such that $u$ and the vectors of $b \cap\left(C_{1} \cup \cdots \cup C_{q}\right)$ are linearly independent. 
But $u \in S$ and $b$ is a basis of $S$. Then we can write $u$ as a linear combination of the vectors $b_{i}$ and such that at least one of the nonzero coefficients is of a vector in $b$ that do not belong to $C_{1} \cup \cdots \cup C_{q}$. Say $b_{i}$. It follows then that $l\left(b_{i}\right)>l(u)$ and so the basis obtained from $b$ by substituting $b_{i}$ by $u,\left\{b_{1}, \ldots, b_{i-1}, u, b_{i+1}, \ldots, b_{r}\right\}$ is such that

$$
l\left(\left\{b_{1}, \ldots, b_{i-1}, u, b_{i+1}, \ldots, b_{r}\right\}\right)<m .
$$

A contradiction.

(ii) $b \subseteq C_{1} \cup \cdots \cup C_{q}$ and there is $j$ between 1 and $q$ such that the number of vectors of $b$ with length lower or equal to $l_{j}$ is (strictly) lower than $\operatorname{dim}\left(S_{1}+\cdots+S_{j}\right)$. Then there is $u \in C_{1} \cup \cdots \cup C_{j}$ such that $u$ and the vectors of $b \cap\left(C_{1} \cup \cdots \cup C_{j}\right)$ are linearly independent. Again, $u$ is a linear combination of the vectors $b_{i}$, and at least one nonzero coefficient corresponds to a vector, say $b_{k}$, such that $l\left(b_{k}\right)>l_{j}$. The basis obtained from $b$ by substituting $b_{k}$ by $u$ has total length strictly lower than $m$. Again a contradiction.

It follows then that any basis $b=\left\{b_{1}, \ldots, b_{r}\right\} \subseteq\left(\left(\mathbb{Z}_{0}^{+}\right)^{k} \cap C\right)$ of the space $S$ such that $l\left(\left\{b_{1}, \ldots, b_{r}\right\}\right)=m$ is contained in $C_{1} \cup \ldots \cup C_{q}$ and the number of vectors of $b$ with length lower or equal to $l_{j}$ is equal to $\operatorname{dim}\left(S_{1}+\cdots+S_{j}\right)$, for $j=1, \ldots, q$. Thus $b=U_{1} \cup \cdots \cup U_{q}$ where the $U_{j}$ are any sets as constructed above. Moreover, we have the equality (6.12).

\section{Algorithm}

Let $G$ be an homogeneous network with $n$ cells and $p-1$ edge-types. Let $M_{(G, l)}=\left[a_{i j}^{l}\right]_{1 \leqslant i, j \leqslant n}$, for $l=1, \ldots, p-1$, be the adjacency matrices associated with $G$ and let $M_{(G, p)}=\mathrm{I}_{n}$. For $l=1, \ldots, p$, let $w_{l}$ be the vector $w_{l}=\left(a_{11}^{l}, \ldots, a_{1 n}^{l}, a_{21}^{l}, \ldots, a_{2 n}^{l}, \ldots, a_{n 1}^{l}, \ldots, a_{n n}^{l}\right)$. Let $k=n^{2}$ and $S$ the real vector subspace of $\mathbb{R}^{k}$ generated by the $p$ vectors $w_{1}, \ldots, w_{p}$ of $\left(\mathbb{Z}_{0}^{+}\right)^{k}$. By theorem 6.7 the following algorithm finds all the minimal bases of $S$ in $\left(\mathbb{Z}_{0}^{+}\right)^{k}$.

In what follows, if $M_{1}$ and $M_{2}$ are matrices of order $m \times n$ and $m \times p$, respectively, we denote by $\left(M_{1} \mid M_{2}\right)$ the matrix of order $m \times(n+p)$ whose first $n$ columns are those of $M_{1}$ and the last $p$ columns are those of $M_{2}$.

Algorithm 7.1. Given $p$ vectors $w_{1}, \ldots, w_{p}$ of $\left(\mathbb{Z}_{0}^{+}\right)^{k}$ that generate a real vector subspace $S$ of $\mathbb{R}^{k}$, this algorithm finds all the minimal bases of $S$ in $\left(\mathbb{Z}_{0}^{+}\right)^{k}$.

(1) [Initial base] Let $r$ be the rank of the matrix $\left(w_{1}|\ldots| w_{p}\right)$. For each $w_{i}, i=1, \ldots, p$ do the following: set $s_{i}=\sum_{j=1}^{k}\left(w_{i}\right)_{j}$ and then sort the vectors $w_{i}$ by ascending order of the $s_{i}$. Let $v_{1}, \ldots, v_{r}$ be the 'first' $r$ linearly independent vectors. Set $s=\sum_{j=1}^{k}\left(v_{r}\right)_{j}$.

(2) [Compute $\operatorname{gcd} \delta$ ] Let $W$ be the matrix $\left(v_{1}|\ldots| v_{r}\right)$. Compute the set $M_{W}$ of all the $r \times r$ matrices formed by $r$ rows of $W$ and with nonzero determinant. Let $\delta$ be the greatest common divisor of the determinants of the matrices in $M_{W}$.

(3) [Solve linear system of congruences] Apply HNF (Hermite Normal Form), algorithm A.3, to the matrix $\left(W \mid \delta \mathrm{I}_{k}\right)$, and let $U=\left(\begin{array}{ll}U_{1} & U_{2} \\ U_{3} & U_{4}\end{array}\right)$ be a unimodular matrix and $H$ the $\mathrm{HNF}$ matrix such that $\left(W \mid \delta \mathrm{I}_{k}\right) U=(0 \mid H)$. Here $U_{1}$ is a $r \times r$ square matrix. We can discard $U_{2}, U_{3}$ and $U_{4}$. Let $H_{B}$ be the HNF matrix of the matrix $\left(U_{1} \mid \delta \mathrm{I}_{r}\right)$ obtained from algorithm A.3. The matrix $H_{B}$ is invertible because it has rank $r$.

(4) [Compute set $C$ ] Let $W^{*}$ be a matrix in $M_{W}$. Determine the set

$$
A=\left\{\left(W^{*} H_{B}\right)^{-1}\left(\delta c_{1}, \delta c_{2}, \ldots, \delta c_{r}\right)^{\top}:\left\{\begin{array}{l}
c_{1}, c_{2}, \ldots, c_{r} \in \mathbb{Z}_{0}^{+} \\
0<c_{1}+c_{2}+\cdots+c_{r} \leqslant s
\end{array}\right\} \cap \mathbb{Z}^{r} .\right.
$$


Determine
$C=\left\{\frac{1}{\delta} W H_{B}\left(x_{1}, \ldots, x_{r}\right)^{\top}:\left(x_{1}, \ldots, x_{r}\right)^{\top} \in A\right\} \cap$
$\left\{w \in(\mathbb{Z} /(s+1) \mathbb{Z})^{k}: 0<l(w) \leqslant s\right\}$.

For $i=1$ until $s$, set $C_{i}=\emptyset$. For each $w$ in $C$ do the following: set $C_{l(w)}=C_{l(w)} \cup\{w\}$.

(5) [Compute minimum sum] Let $j$ be the least $i$ such that $C_{i} \neq \emptyset$. Set $m=0, i=j$ and $d=0$. While $i \leqslant s$ and $d<r$ do the following: if $C_{i} \neq \emptyset$, let $d_{i}$ be the dimension of the real subspace generated by $C_{j} \cup \cdots \cup C_{i}$ minus the dimension of the real subspace generated by $C_{j} \cup \cdots \cup C_{i-1}$ and set $d=d+d_{i}, m=m+i d_{i}$, else set $d_{i}=0$; set $i=i+1$.

(6) [All subsets of $C$ with minimum sum] Set $E=\emptyset, q=0$ and $S_{j-1}=\{0\}$. For $n=j$ until $i-1$ do the following: if $d_{n} \neq 0$, set $I_{n}=\left\{w \in C_{n}: w \notin S_{j}+\cdots+S_{n-1}\right\}$ and let $E_{n}$ be the set of all the subsets of $I_{n}$ with $d_{n}$ linearly independent vectors and set $E=E \times E_{n}$ and $q=q+1$.

(7) [All minimal bases] Set $U=\emptyset$. For each $\left(U_{1}, \ldots, U_{q}\right)$ in $E$ do the following: if the dimension of the subspace generated by $U_{1} \cup \cdots \cup U_{q}$ is $r$ (that is, $U_{1} \cup \cdots \cup U_{q}$ is a minimal base of $S$ in $\left.\left(\mathbb{Z}_{0}^{+}\right)^{k}\right)$ then set $U=U \cup\left\{\left\{U_{1} \cup \cdots \cup U_{q}\right\}\right\}$.

The set $U$ contains all the minimal bases of $S$ in $\left(\mathbb{Z}_{0}^{+}\right)^{k}$. Output $U$ and $m$ and terminate algorithm.

Item (3) of algorithm 7.1 for solving homogeneous linear systems of congruences is based on Cohen [3, algorithm 4.1.22].

Each basis $B=\left\{b_{1}, \ldots, b_{r}\right\} \in U$, computed by algorithm 7.1, corresponds to a minimal network with $r-1$ edge-types in the following way: Each $b_{i} \in\left(\mathbb{Z}_{0}^{+}\right)^{k}$ defines an $n \times n$ matrix $M_{i}$. Specifically, if $b_{i}=\left(b_{i 1}, \ldots, b_{i n}\right)$ where $b_{i j} \in\left(\mathbb{Z}_{0}^{+}\right)^{n}$ then the $j$ th row of $M_{i}$ is $b_{i j}$. Each matrix $M_{i} \neq \mathrm{I}_{n}$ corresponds to an adjacency matrix of the minimal network with respect to an edge-type.

\section{Examples}

In this section we apply algorithm 7.1 to two examples of homogeneous coupled cell networks $G$ obtaining the adjacency matrices corresponding to the minimal networks of the subclasses $\mathcal{M i n}_{[G]}$ of $[G]$.

Example 8.1. Consider the homogeneous network $G$ of three cells of figure 7. Thus the adjacency matrices are

$$
M_{(G, 1)}=\left(\begin{array}{ccc}
4 & 0 & 6 \\
8 & 0 & 2 \\
3 & 2 & 5
\end{array}\right), \quad M_{(G, 2)}=\left(\begin{array}{rrr}
4 & 8 & 4 \\
8 & 4 & 4 \\
10 & 4 & 2
\end{array}\right) .
$$

Note that

$$
\operatorname{dim} \mathbb{R}\left\{\mathrm{I}_{3}, M_{(G, 1)}, M_{(G, 2)}\right\}=3
$$

and recall that the adjacency matrices of the networks in $\mathcal{M i n}_{[G]}$ satisfy the three properties of remark 5.12. Thus, our aim is to find all the possible matrices $M_{1}, M_{2} \in M_{3 \times 3}\left(\mathbb{Z}_{0}^{+}\right)$such that $\mathbb{R}\left\{M_{0}=\mathrm{I}_{3}, M_{1}, M_{2}\right\}=\mathbb{R}\left\{\mathrm{I}_{3}, M_{(G, 1)}, M_{(G, 2)}\right\}$ and

$\sum_{k=0}^{2} l\left(M_{k}\right)=\min \left\{\sum_{k=0}^{2} l\left(b_{k}\right):\left\{b_{0}=\mathrm{I}_{3}, b_{1}, b_{2}\right\} \subset M_{3 \times 3}\left(\mathbb{Z}_{0}^{+}\right)\right.$

is a basis of $\left.\mathbb{R}\left\{\mathrm{I}_{3}, M_{(G, 1)}, M_{(G, 2)}\right\}\right\}$. 


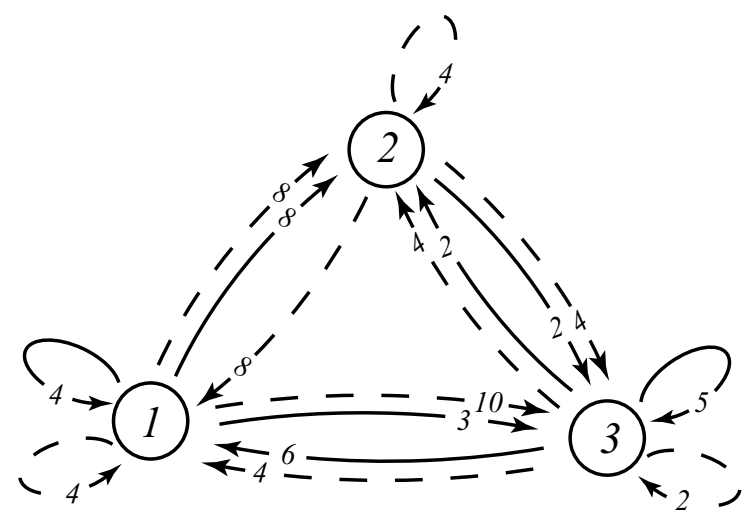

Figure 7. Homogeneous coupled cell network of example 8.1.

For that we apply algorithm 7.1. We consider

$$
\begin{gathered}
w_{1}=(1,0,0,0,1,0,0,0,1)^{\top}, \quad w_{2}=(4,0,6,8,0,2,3,2,5)^{\top}, \\
w_{3}=(4,8,4,8,4,4,10,4,2)^{\top}
\end{gathered}
$$

in $\left(\mathbb{Z}_{0}^{+}\right)^{9}$ and $S$ the real subspace of $\mathbb{R}^{9}$ generated by these linearly independent vectors:

$$
S=\mathbb{R}\left\{w_{1}, w_{2}, w_{3}\right\}
$$

Note that

$$
s=\max \left\{l\left(w_{1}\right), l\left(w_{2}\right), l\left(w_{3}\right)\right\}=l\left(w_{3}\right)=48
$$

and the matrix

$$
W^{\top}=\left(\begin{array}{rrrrrrrrr}
1 & 0 & 0 & 0 & 1 & 0 & 0 & 0 & 1 \\
4 & 0 & 6 & 8 & 0 & 2 & 3 & 2 & 5 \\
4 & 8 & 4 & 8 & 4 & 4 & 10 & 4 & 2
\end{array}\right)
$$

has rank 3. The greatest common divisor of the determinants of all $3 \times 3$ matrices formed by 3 rows of $W$ and with nonzero determinant is $\delta=8$. Applying the HNF algorithm A.3 to the matrix $\left(W \mid 8 \mathrm{I}_{9}\right)$ we obtain matrices $U$ and $H$, the HNF of $\left(W \mid 8 \mathrm{I}_{9}\right)$, such that $\left(W \mid 8 \mathrm{I}_{9}\right) U=$ $(0 \mid H)$, where

$$
U=\left(\begin{array}{lll}
U_{1} & U_{2} & U_{3} \\
U_{4} & U_{5} & U_{6} \\
U_{7} & U_{8} & U_{9}
\end{array}\right)
$$

with

$$
\begin{gathered}
U_{1}=\left(\begin{array}{rrr}
-8 & 44 & -16 \\
0 & -10 & 4 \\
0 & 3 & -2
\end{array}\right), \quad U_{3}=\left(\begin{array}{rrrrr}
8 & -5 & 1 \\
-2 & 1 & 0 \\
1 & 0 & 0
\end{array}\right), \\
U_{4}^{\top}=\left(\begin{array}{rrrrrr}
1 & 0 & 0 & 0 & 1 & 0 \\
-2 & -3 & 6 & 7 & -7 & 1 \\
1 & 2 & -2 & -2 & 3 & 0
\end{array}\right),
\end{gathered}
$$


$U_{6}^{\top}=\left(\begin{array}{rrrrrr}0 & -1 & 1 & 1 & -1 & 0 \\ 1 & 0 & 0 & -1 & 1 & 0 \\ 0 & 0 & 0 & 0 & 0 & 0\end{array}\right), \quad H=\left(\begin{array}{rrrrrrrrr}8 & 0 & 0 & 0 & 0 & 0 & 4 & 7 & 1 \\ 0 & 8 & 0 & 0 & 0 & 0 & 0 & 0 & 0 \\ 0 & 0 & 8 & 0 & 0 & 0 & 0 & 6 & 0 \\ 0 & 0 & 0 & 8 & 0 & 0 & 0 & 0 & 0 \\ 0 & 0 & 0 & 0 & 8 & 0 & 4 & 3 & 1 \\ 0 & 0 & 0 & 0 & 0 & 8 & 0 & 2 & 0 \\ 0 & 0 & 0 & 0 & 0 & 0 & 4 & 3 & 0 \\ 0 & 0 & 0 & 0 & 0 & 0 & 0 & 2 & 0 \\ 0 & 0 & 0 & 0 & 0 & 0 & 0 & 0 & 1\end{array}\right)$,

$U_{7}^{\top}=\mathrm{I}_{3}, U_{5}=\mathrm{I}_{6}$, and $U_{2}, U_{8}$ and $U_{9}$ are zero matrices.

Using algorithm A.3 to compute $H_{B}$, the HNF of the matrix $\left(U_{1} \mid 8 \mathrm{I}_{3}\right)$, we obtain

$H_{B}=\left(\begin{array}{rrr}8 & 0 & 4 \\ 0 & 8 & 2 \\ 0 & 0 & 1\end{array}\right) \quad$ and $\quad\left(W H_{B}\right)^{\top}=\left(\begin{array}{rrrrrrrrr}8 & 0 & 0 & 0 & 8 & 0 & 0 & 0 & 8 \\ 32 & 0 & 48 & 64 & 0 & 16 & 24 & 16 & 40 \\ 16 & 8 & 16 & 24 & 8 & 8 & 16 & 8 & 16\end{array}\right)$

and so

$C=\left\{\left(\begin{array}{c}x_{1}+4 x_{2}+2 x_{3} \\ x_{3} \\ 6 x_{2}+2 x_{3} \\ 8 x_{2}+3 x_{3} \\ x_{1}+x_{3} \\ 2 x_{2}+x_{3} \\ 3 x_{2}+2 x_{3} \\ 2 x_{2}+x_{3} \\ x_{1}+5 x_{2}+2 x_{3}\end{array}\right) \in\left(\mathbb{Z}_{0}^{+}\right)^{9}: x_{1}, x_{2}, x_{3} \in \mathbb{Z}, 0<x_{1}+10 x_{2}+5 x_{3} \leqslant 16\right\}$.

In particular, it follows that for

$w=\left(x_{1}+4 x_{2}+2 x_{3}, x_{3}, 6 x_{2}+2 x_{3}, 8 x_{2}+3 x_{3}, x_{1}+x_{3}, 2 x_{2}+x_{3}, 3 x_{2}+2 x_{3}\right.$,

$$
\left.2 x_{2}+x_{3}, x_{1}+5 x_{2}+2 x_{3}\right)^{\top} \in C
$$

we have $l(w) \geqslant 3 x_{3}$ and $x_{3} \geqslant 0$. Easy computations show that

$C_{3}=\left\{\left(\begin{array}{l}1 \\ 0 \\ 0 \\ 0 \\ 1 \\ 0 \\ 0 \\ 0 \\ 1\end{array}\right), \quad C_{6}=\left\{\left(\begin{array}{l}2 \\ 0 \\ 0 \\ 0 \\ 2 \\ 0 \\ 0 \\ 0 \\ 2\end{array}\right), \quad C_{9}=\left\{\left(\begin{array}{l}3 \\ 0 \\ 0 \\ 0 \\ 3 \\ 0 \\ 0 \\ 0 \\ 3\end{array}\right), \quad C_{12}=\left\{\left(\begin{array}{l}4 \\ 0 \\ 0 \\ 0 \\ 0 \\ 0 \\ 0 \\ 4\end{array}\right),\left(\begin{array}{l}1 \\ 1 \\ 2 \\ 3 \\ 0 \\ 1 \\ 2 \\ 1 \\ 1\end{array}\right),\left(\begin{array}{l}1 \\ 3 \\ 0 \\ 1 \\ 2 \\ 1 \\ 0\end{array}\right)\right.\right.\right.\right.$.

Moreover, if $S_{i}$ denotes the real subspace of $S$ generated by $C_{i}$, we have that

$\operatorname{dim} S_{3}=\operatorname{dim}\left(S_{3}+S_{6}\right)=\operatorname{dim}\left(S_{3}+S_{6}+S_{9}\right)=1, \quad \operatorname{dim}\left(S_{3}+S_{6}+S_{9}+S_{12}\right)=\operatorname{dim} S=3$ 


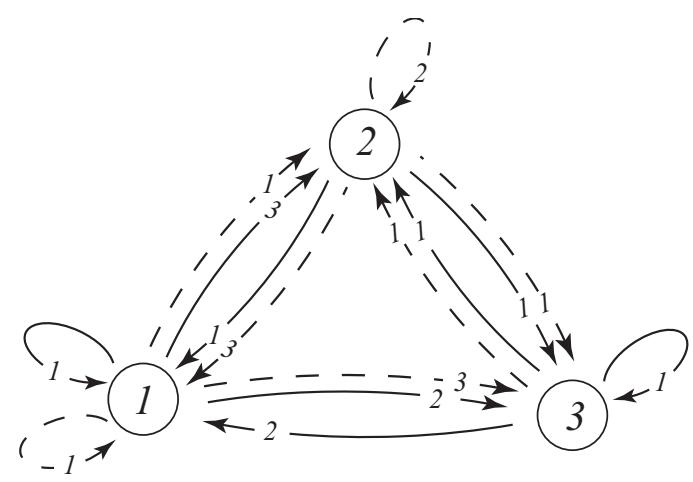

Figure 8. Minimal network of the ODE-equivalence class of the homogeneous coupled cell network of example 8.1 .

By theorem 6.7, there is a unique minimal basis of $S$ which is

$$
\left\{\left(\begin{array}{l}
1 \\
0 \\
0 \\
0 \\
1 \\
0 \\
0 \\
0 \\
1
\end{array}\right),\left(\begin{array}{l}
1 \\
1 \\
2 \\
3 \\
0 \\
1 \\
2 \\
1 \\
1
\end{array}\right),\left(\begin{array}{l}
1 \\
3 \\
0 \\
1 \\
2 \\
1 \\
3 \\
1 \\
0
\end{array}\right)\right\} \text {. }
$$

Thus, by proposition 5.11 and remark $5.12, \mathcal{M i n}_{[G]}$ contains only one minimal network with adjacency matrices given by

$$
M_{1}=\left(\begin{array}{lll}
1 & 1 & 2 \\
3 & 0 & 1 \\
2 & 1 & 1
\end{array}\right), \quad M_{2}=\left(\begin{array}{lll}
1 & 3 & 0 \\
1 & 2 & 1 \\
3 & 1 & 0
\end{array}\right)
$$

See figure 8 .

Example 8.2. Consider the homogeneous network $G$ of figure 3 in section 1 . The adjacency matrices of $G$ are

$M_{(G, 1)}=\left(\begin{array}{lll}1 & 1 & 2 \\ 2 & 1 & 1 \\ 3 & 0 & 1\end{array}\right), \quad M_{(G, 2)}=\left(\begin{array}{lll}1 & 3 & 0 \\ 0 & 1 & 3 \\ 1 & 2 & 1\end{array}\right), \quad$ and $\operatorname{dim} \mathbb{R}\left\{I_{3}, M_{(G, 1)}, M_{(G, 2)}\right\}=3$.

We aim to find all the possible matrices $M_{1}, M_{2} \in M_{3 \times 3}\left(\mathbb{Z}_{0}^{+}\right)$such that $\mathbb{R}\left\{M_{0}=\mathrm{I}_{3}, M_{1}, M_{2}\right\}=$ $\mathbb{R}\left\{\mathrm{I}_{3}, M_{(G, 1)}, M_{(G, 2)}\right\}$ and such that

$$
\begin{gathered}
\sum_{k=0}^{2} l\left(M_{k}\right)=\min \left\{\sum_{k=0}^{2} l\left(b_{k}\right):\left\{b_{0}=\mathrm{I}_{3}, b_{1}, b_{2}\right\} \subset M_{3 \times 3}\left(\mathbb{Z}_{0}^{+}\right)\right. \\
\text {is a basis of } \left.\mathbb{R}\left\{\mathrm{I}_{3}, M_{(G, 1)}, M_{(G, 2)}\right\}\right\} .
\end{gathered}
$$


Again, we apply algorithm 7.1 to the vectors

$$
\begin{gathered}
w_{1}=(1,0,0,0,1,0,0,0,1)^{\top}, \quad w_{2}=(1,1,2,2,1,1,3,0,1)^{\top}, \\
w_{3}=(1,3,0,0,1,3,1,2,1)^{\top}
\end{gathered}
$$

in $\left(\mathbb{Z}_{0}^{+}\right)^{9}$. Note that $s=l\left(w_{3}\right)=12$. Easy computations show that

$$
C=\left\{\left(\begin{array}{c}
x_{1}+x_{2}+x_{3} \\
x_{2}+2 x_{3} \\
2 x_{2}+x_{3} \\
2 x_{2}+x_{3} \\
x_{1}+x_{2}+x_{3} \\
x_{2}+2 x_{3} \\
3 x_{2}+2 x_{3} \\
x_{3} \\
x_{1}+x_{2}+x_{3}
\end{array}\right) \in\left(\mathbb{Z}_{0}^{+}\right)^{9}: x_{1}, x_{2}, x_{3} \in \mathbb{Z}, 0<x_{1}+4 x_{2}+4 x_{3} \leqslant 4\right\} .
$$

Moreover,

$$
C_{3}=\left\{\left(\begin{array}{l}
1 \\
0 \\
0 \\
0 \\
1 \\
0 \\
0 \\
0 \\
1
\end{array}\right), C_{6}=\left\{\left(\begin{array}{l}
2 \\
0 \\
0 \\
0 \\
2 \\
0 \\
0 \\
0 \\
2
\end{array}\right), C_{9}=\left\{\left(\begin{array}{l}
3 \\
0 \\
0 \\
0 \\
3 \\
0 \\
0 \\
0 \\
3
\end{array}\right),\left(\begin{array}{l}
0 \\
2 \\
1 \\
1 \\
0 \\
2 \\
2 \\
1 \\
0
\end{array}\right),\left(\begin{array}{l}
0 \\
1 \\
2 \\
2 \\
0 \\
1 \\
3 \\
0 \\
0
\end{array}\right),\left(\begin{array}{l}
0 \\
3 \\
0 \\
0 \\
0 \\
3 \\
1 \\
2 \\
0
\end{array}\right)\right\} .\right.\right.
$$

If $S_{i}$ denotes the real subspace of $S$ generated by $C_{i}$, we have that

$$
\operatorname{dim} S_{3}=\operatorname{dim}\left(S_{3}+S_{6}\right)=1, \quad \operatorname{dim}\left(S_{3}+S_{6}+S_{9}\right)=\operatorname{dim} S=3 .
$$

Thus, by proposition 5.11 and remark 5.12, $\mathcal{M i n}_{[G]}$ contains three minimal networks with adjacency matrices given, respectively, by

$$
\begin{gathered}
\left\{M_{1}=\left(\begin{array}{lll}
0 & 2 & 1 \\
1 & 0 & 2 \\
2 & 1 & 0
\end{array}\right), M_{2}=\left(\begin{array}{lll}
0 & 1 & 2 \\
2 & 0 & 1 \\
3 & 0 & 0
\end{array}\right)\right\}, \\
\left\{M_{1}=\left(\begin{array}{lll}
0 & 2 & 1 \\
1 & 0 & 2 \\
2 & 1 & 0
\end{array}\right), M_{2}=\left(\begin{array}{lll}
0 & 3 & 0 \\
0 & 0 & 3 \\
1 & 2 & 0
\end{array}\right)\right\}
\end{gathered}
$$

and

$$
\left\{M_{1}=\left(\begin{array}{lll}
0 & 1 & 2 \\
2 & 0 & 1 \\
3 & 0 & 0
\end{array}\right), M_{2}=\left(\begin{array}{lll}
0 & 3 & 0 \\
0 & 0 & 3 \\
1 & 2 & 0
\end{array}\right)\right\} .
$$

See figure 4 in section 1. 


\section{Identical-edge homogeneous networks}

In the special class of identical-edge homogeneous cell networks, the application of algorithm 7.1 simplifies. Recall that an identical-edge homogeneous cell network is an homogeneous network in which all edges in $\mathcal{E}$ are equivalent. We prove below (theorem 9.3) that, if $G$ is an identical-edge homogeneous network with $n$ cells, with adjacency matrix $M_{(G, 1)}=\left[a_{i j}\right]_{1 \leqslant i, j \leqslant n}$, and $[G]$ the corresponding ODE-class, then $\mathcal{M i n}_{[G]}$ has a unique graph with adjacency matrix $M$ defined by

$$
M=\frac{1}{\delta}\left(M_{(G, 1)}-m \mathrm{I}_{n}\right),
$$

where $\delta=\operatorname{gcd}\left\{a_{i j}: i, j=1, \ldots, n ; j \neq i\right\}$ and $m=\min \left\{a_{i i}: i=1, \ldots, n\right\}$. Direct application of algorithm 7.1 gives the same result. See remark 9.5. Here, we consider only networks $G$ such that there is at least one edge $e \in \mathcal{E}$ satisfying $\mathcal{H}(e) \neq \mathcal{T}(e)$, and so $M_{(G, 1)}$ is not a scalar multiple of the identity matrix.

Example 9.1. The graphs $G_{1}$ and $G_{2}$ of figures 1 and 2 in section 1 , respectively, are examples of identical-edge homogeneous coupled cell networks. The corresponding adjacency matrices are

$$
M_{\left(G_{1}, 1\right)}=\left(\begin{array}{rrr}
2 & 9 & 9 \\
12 & 2 & 6 \\
15 & 0 & 5
\end{array}\right), \quad M_{\left(G_{2}, 1\right)}=\left(\begin{array}{ccc}
0 & 3 & 3 \\
4 & 0 & 2 \\
5 & 0 & 1
\end{array}\right)
$$

We show below that the minimal subclass of $\left[G_{1}\right]$ is $\mathcal{M i n}_{\left[G_{1}\right]}=\left\{G_{2}\right\}$.

\subsection{Linear equivalence and adjacency matrices}

Definition 3.2 of linear equivalence for identical-edge homogeneous networks in terms of the adjacency matrices is now a special case of proposition 5.4. More precisely, if $G_{1}$ and $G_{2}$ are two identical-edge homogeneous networks with $n$ cells, where $\mathcal{C}_{1}=\{1, \ldots, n\}$, and $M_{\left(G_{1}, 1\right)}=\left[a_{i j}\right]_{1 \leqslant i, j \leqslant n}$ and $M_{\left(G_{2}, 2\right)}=\left[b_{i j}\right]_{1 \leqslant i, j \leqslant n}$ are the corresponding adjacency matrices, then $G_{1}$ and $G_{2}$ are linearly equivalent if and only if there is a bijection $\gamma: \mathcal{C}_{1} \rightarrow \mathcal{C}_{2}$ such that:

$$
\mathbb{R}\left\{\operatorname{Id}_{n},\left[a_{i j}\right]_{1 \leqslant i, j \leqslant n}\right\}=\mathbb{R}\left\{\operatorname{Id}_{n},\left[b_{\gamma(i) \gamma(j)}\right]_{1 \leqslant i, j \leqslant n}\right\} .
$$

Example 9.2. Consider the identical-edge homogeneous networks of figures 1 and 2 in section 1, and recall example 3.4. Trivially, the identity function on $\{1,2,3\}$ is a bijection that preserves cell-equivalence and input-equivalence (like any bijection on $\{1,2,3\}$ ). Moreover, equation (3.4) of example 3.4 on the corresponding adjacency matrices is equivalent to the linear equivalence of the networks.

\subsection{Minimality}

We prove now that the minimal subclass $\mathcal{M i n}_{[G]}$ of an ODE-class $[G]$ where $G$ is an identicaledge homogeneous network contains a unique graph. We then describe an algorithm that computes this minimal graph in $\mathcal{M i n}_{[G]}$.

Theorem 9.3. Let $G$ be an identical-edge homogeneous network of n cells, say $\mathcal{C}=\{1, \ldots, n\}$, and $[G]$ the corresponding ODE-class. Let $M_{(G, 1)}=\left[a_{i j}\right]_{1 \leqslant i, j \leqslant n}$ be the adjacency matrix of $G$ and assume that it is not a scalar multiple of the identity matrix. Let

$$
\begin{aligned}
& \delta=\operatorname{gcd}\left\{a_{i j}: i, j=1, \ldots, n ; j \neq i\right\}, \\
& m=\min \left\{a_{i i}: i=1, \ldots, n\right\} .
\end{aligned}
$$


Then

$$
\mathcal{M i n}_{[G]}=\left\{G_{2}\right\},
$$

where $G_{2}$ is the network with adjacency matrix

$$
M_{\left(G_{2}, 1\right)}=\frac{1}{\delta}\left(M_{(G, 1)}-m \mathrm{I}_{n}\right) .
$$

Proof. The proof consists in showing that $G_{2}$ as described above is linearly equivalent to $G$, and that if $G_{1}$ is linearly equivalent to $G_{2}$ and $G_{1} \neq G_{2}$ then $\operatorname{card}\left(\mathcal{E}_{2}\right)<\operatorname{card}\left(\mathcal{E}_{1}\right)$.

As $M_{\left(G_{2}, 1\right)}=(1 / \delta)\left(M_{(G, 1)}-m \mathrm{I}_{n}\right)$ it follows that the identical-edge homogeneous network with adjacency matrix $M_{\left(G_{2}, 1\right)}$ is linearly equivalent to $G$ and the entries of $M_{\left(G_{2}, 1\right)}$ are nonnegative integers as we show now. Let $M_{\left(G_{2}, 1\right)}=\left[b_{i j}\right]_{1 \leqslant i, j \leqslant n}$, then

$$
\begin{aligned}
& b_{i i}=\frac{a_{i i}-m}{\delta}, i=1, \ldots, n \\
& b_{i j}=\frac{a_{i j}}{\delta}, i, j=1, \ldots, n ; j \neq i .
\end{aligned}
$$

Since $G$ is an identical-edge homogeneous network it follows that $M_{(G, 1)}$ satisfies for any $i, k \in\{1, \ldots, n\}$

$$
\sum_{j=1}^{n} a_{i j}=\sum_{j=1}^{n} a_{k j}
$$

and thus

$$
a_{i i}-a_{k k}=\sum_{j \neq k} a_{k j}-\sum_{j \neq i} a_{i j} .
$$

Let $k$ be such that $m=a_{k k}$. Then $a_{k k} \leqslant a_{i i}, \forall i$ and $\sum_{j \neq k} a_{k j} \geqslant \sum_{j \neq i} a_{i j}$. Moreover, $\delta$ is a divisor of $\sum_{j \neq k} a_{k j}-\sum_{j \neq i} a_{i j}$ and thus of $a_{i i}-a_{k k}$.

Let $G_{1}$ be a network linearly equivalent to $G_{2}$ (and so to $G$ ) with adjacency matrix $M_{\left(G_{1}, 1\right)}$. It follows then that

$$
M_{\left(G_{1}, 1\right)}=\frac{1}{\beta}\left(M_{\left(G_{2}, 1\right)}-\alpha \mathrm{I}_{n}\right)
$$

for some $\alpha \in \mathbb{Q}$ and $\beta \in \mathbb{Q}^{+}$. Since there is $i$ such that $b_{i i}=0$ we have that $\alpha \in \mathbb{Z}_{0}^{-}$. If $\operatorname{card}\left(\mathcal{E}_{1}\right) \leqslant \operatorname{card}\left(\mathcal{E}_{2}\right)$ then $\beta \geqslant 1$. Since the greatest common divisor of the entries $b_{i j}$ is 1 , then $\beta=1$. If $\alpha=0$ then $G_{1}=G_{2}$. Otherwise, $\operatorname{card}\left(\mathcal{E}_{1}\right)>\operatorname{card}\left(\mathcal{E}_{2}\right)$.

Example 9.4. Recall the graphs $G_{1}$ and $G_{2}$ of figures 1 and 2 in section 1 . By theorem 9.3 it follows that $\mathcal{M i n}_{G_{1}}=\left\{G_{2}\right\}$. Note that $\delta=3$ and $m=2$.

If we denote the rows of a $k \times r$ matrix $M$ by $M_{1}, \ldots, M_{k}$, then $M^{*}$ represents the column vector $\left(M_{1}, \ldots, M_{k}\right)^{\top}$.

Remark 9.5. Direct application of algorithm 7.1 gives the result of theorem 9.3 as we now show. Suppose $G$ is an identical-edge homogeneous network with adjacency matrix $M_{(G, 1)}=\left[a_{i j}\right]_{1 \leqslant i, j \leqslant n}$. Assume $M_{(G, 1)}$ is not a scalar multiple of the identity matrix. Using the same procedure as in section 6 we have the following: we take the $n^{2} \times 2$ matrix $W=\left(w_{1} \mid w_{2}\right)$ where $w_{1}=\mathrm{I}_{n}^{*}$ and $w_{2}=M_{(G, 1)}^{*}$; the greatest common divisor $\delta$ of the set of all $2 \times 2$ matrices formed by 2 rows of $W$ and with nonzero determinant is the greatest common divisor of the set $\left\{a_{i j}: i \neq j\right\} \cup\left\{a_{i i}-a_{k k}: i \neq k\right\}$. From the proof of theorem 9.3, it follows that $\delta$ is the 
greatest common divisor of the set $\left\{a_{i j}: i \neq j\right\}$. We aim to find the finite set $C$ and so to find $y_{1}, y_{2} \in \mathbb{Z}$ such that

$$
y_{1} w_{1}+y_{2} w_{2}=\delta w
$$

for some $w$ with non-negative integer entries and such that $0<l(w) \leqslant l\left(w_{2}\right)$. Note that

$$
l(w)=\frac{1}{\delta}\left(y_{1} n+y_{2} l\left(w_{2}\right)\right)
$$

and

$$
\begin{aligned}
& \delta w_{(k-1) n+k}=y_{1}+y_{2} a_{k k} \quad(k=1, \ldots, n), \\
& \delta w_{(k-1) n+i}=y_{2} a_{k i} \quad(i, k=1, \ldots, n ; \quad i \neq k) .
\end{aligned}
$$

Thus $y_{2} \geqslant 1$. Trivially, the minimum $l(w)$ is obtained by taking $y_{2}=1$ and $y_{1}=-m$ where

$$
m=\min \left\{a_{k k}: k=1, \ldots, n\right\}
$$

and so there is a unique minimal basis of $\mathbb{R}\left\{w_{1}, w_{2}\right\}$ corresponding to the minimal network with adjacency matrix

$$
M_{\left(G_{2}, 1\right)}=\frac{1}{\delta}\left(M_{(G, 1)}-m \mathrm{I}_{n}\right) .
$$

Algorithm 9.6. Given the adjacency matrix $M_{(G, 1)}=\left[a_{i j}\right]_{1 \leqslant i, j \leqslant n}$, with $M_{(G, 1)} \neq \alpha \mathrm{I}_{n}$, of an identical-edge homogeneous network $G$, this algorithm computes the adjacency matrix $M_{\left(G_{2}, 1\right)}=\left[b_{i j}\right]_{1 \leqslant i, j \leqslant n}$ corresponding to the unique network in $\mathcal{M i n}_{[G]}$.

(1) [Compute $\operatorname{gcd} \delta$ and minimum $m]$ Set $m=\min \left\{a_{i i}: i=1, \ldots, n\right\}$ and $\delta=\operatorname{gcd}\left\{a_{i j}: i, j=1, \ldots, n ; j \neq i\right\}$.

(2) [Compute $M_{\left(G_{2}, 1\right)}$ ] For $i, j=1, \cdots, n$ do the following: if $j \neq i$ then set $b_{i j}=a_{i j} / \delta$ else set $b_{i i}=\left(a_{i i}-m\right) / \delta$. Output $M_{\left(G_{2}, 1\right)}=\left[b_{i j}\right]_{1 \leqslant i, j \leqslant n}$.

\section{Minimality for coupled cell networks}

Let $G$ be a coupled cell network. From theorem 2.3 (recall also remark 2.5) the space of $\mathcal{B}_{G}$-equivariant maps has a natural decomposition according to the connected components (the $\sim_{I}$-equivalence classes) of the groupoid $\mathcal{B}_{G}$, and this decomposition is inherited by the polynomial and linear vector fields:

Definition 10.1. Let $\mathcal{Q} \subseteq \mathcal{C}$ be an $\sim_{I}$-equivalence class. Define

$$
\begin{aligned}
& \mathcal{F}_{G}^{P}(\mathcal{Q})=\left\{f \in \mathcal{F}_{G}^{P}: f_{s}(x)=0, \forall s \notin \mathcal{Q}\right\}, \\
& \mathcal{P}_{G}^{P}(\mathcal{Q})=\left\{f \in \mathcal{P}_{G}^{P}: f_{s}(x)=0, \forall s \notin \mathcal{Q}\right\}, \\
& \mathcal{L}_{G}^{P}(\mathcal{Q})=\left\{f \in \mathcal{L}_{G}^{P}: f_{s}(x)=0, \forall s \notin \mathcal{Q}\right\} .
\end{aligned}
$$

We say that vector fields in $\mathcal{F}_{G}^{P}(\mathcal{Q}), \mathcal{P}_{G}^{P}(\mathcal{Q})$, and $\mathcal{L}_{G}^{P}(\mathcal{Q})$ are supported on $\mathcal{Q}$.

Remark 10.2. From theorem 2.3 , there are direct sum decompositions

$$
\mathcal{F}_{G}^{P}=\bigoplus_{\mathcal{Q}} \mathcal{F}_{G}^{P}(\mathcal{Q}) \quad \mathcal{P}_{G}^{P}=\bigoplus_{\mathcal{Q}} \mathcal{P}_{G}^{P}(\mathcal{Q}) \quad \mathcal{L}_{G}^{P}=\bigoplus_{\mathcal{Q}} \mathcal{L}_{G}^{P}(\mathcal{Q}),
$$

where $\mathcal{Q}$ runs over the $\sim_{I}$-equivalence classes of $G$.

For detailed proofs see [9], end of section 4, especially proposition 4.6. 
By theorem 3.5, two coupled cell networks $G_{1}$ and $G_{2}$ are ODE-equivalent if and only if they are linearly equivalent. That is, there exists some bijection $\gamma$ between the corresponding sets of cells $\mathcal{C}_{i}$, preserving cell-equivalence and input-equivalence, such that with the identification $\gamma: \mathcal{C}_{1} \rightarrow \mathcal{C}_{2}$, the vector spaces $\mathcal{L}_{G_{1}}^{P}$ and $\mathcal{L}_{G_{2}}^{P}$ are equal. By the above discussion, this is equivalent to have $\mathcal{L}_{G_{1}}^{P}(\mathcal{Q})=\mathcal{L}_{G_{2}}^{P}(\mathcal{Q})$, for each connected component $\mathcal{Q}$. Moreover, all cell phase spaces may be assumed to be $\mathbb{R}$ in this context.

We look now for $\mathcal{M i n}_{[G]}$ where $G$ is an $n$-cell network. Suppose that $G$ has connected components $\left(\sim_{I}\right.$-equivalence classes $) \mathcal{Q}_{1}, \ldots, \mathcal{Q}_{l}$. For $i=1, \ldots, l$ define the subnetworks $G_{i}$ in the following way:

$$
G_{i}=\left\{\mathcal{C}, \mathcal{E}_{i}, \sim_{C}, \sim_{E_{i}}\right)
$$

where

$$
\mathcal{E}_{i}=\left\{e \in \mathcal{E}: \mathcal{H}(e) \in \mathcal{Q}_{i}\right\} \quad \text { and } \forall e_{1}, e_{2} \in \mathcal{E}_{i}, e_{1} \sim_{E_{i}} e_{2} \Leftrightarrow e_{1} \sim_{E} e_{2} .
$$

We can apply algorithm 7.1 to the graphs $G_{i}$, for $i=1, \ldots, l$, computing $\mathcal{M i n}_{\left[G_{1}\right]}, \ldots$, $\mathcal{M i n}_{\left[G_{l}\right]}$. The minimal class $\mathcal{M i n}_{[G]}$ is then obtained by considering all possible graphs that can be formed by junction of one graph of each class $\mathcal{M i n} n_{\left[G_{1}\right]}, \ldots, \mathcal{M i n}_{\left[G_{l}\right]}$.

\section{Summary}

Non-isomorphic coupled cell networks with the same number of cells can generate the same dynamical behaviour and are called ODE-equivalent. Given an arbitrary number $k$ of cells, we can partition the set of the $k$-cell networks into ODE-equivalence classes.

Given an ODE-class, we can ask for the network, or the networks, in the class with minimal number of edges. We start by addressing the case where the networks are homogeneous, that is, where all the cells are isomorphic. We prove that, in the special case of an ODE-class including an identical-edge homogenous network, there is a unique minimal network (theorem 9.3) which can be obtained using algorithm 9.6.

For general homogeneous networks, there can be more than one minimal network (theorem 6.7) which can be computed using algorithm 7.1. In section 8, we illustrate the application of the algorithm with two examples.

We end by remarking that the minimality of a non-homogeneous network reduces to the minimality of certain homogeneous subnetworks.

\section{Appendix A. Algorithms}

In this section we present an algorithm given by Cohen [2] to calculate the Hermite normal form of a matrix with integer coefficients. We use the following definition:

Definition A.1 ([2], definition 2.4.2). We say that an $m \times n$ matrix $M=\left[m_{i j}\right]$ with integer coefficients is in Hermite normal form (abbreviated HNF) if there exists $r \leqslant n$ and a strictly increasing map $f$ from $[r+1, n]$ to $[1, m]$ satisfying the following properties:

(1) For $r+1 \leqslant j \leqslant n, m_{f(j) j} \geqslant 1, m_{i j}=0$ if $i>f(j)$ and $0 \leqslant m_{f(k) j}<m_{f(k) k}$ if $k<j$.

(2) The first $r$ columns of $M$ are equal to 0 .

We have the following result:

Theorem A.2 ([2], theorem 2.4.3). Let $A$ be an $m \times n$ matrix with coefficients in $\mathbb{Z}$. Then there exists a unique $m \times n$ matrix $B=\left[b_{i j}\right]$ in $H N F$ of the form $B=A U$ with $U \in G L_{n}(\mathbb{Z})$, where $G L_{n}(\mathbb{Z})$ is the group of matrices with integer coefficients which are invertible, that is, whose determinant is equal to \pm 1 . 
Although $B$ is unique, the matrix $U$ is not unique. The matrix $W$ formed by the nonzero columns of $B$ is called the Hermite normal form of the matrix $A$.

Algorithm A.3 ([2], algorithm 2.4.4). (Hermite normal form). Given an $m \times n$ matrix $A$ with integer coefficients $\left[a_{i j}\right]$ this algorithm finds the Hermite normal form $H$ of $A$ and a $n \times n$ unimodular matrix $U$ such that $A U=(0 \mid H)$. We write $h_{i j}$ for the coefficients of $H, A_{i}$ (resp. $\left.H_{i}, U_{i}\right)$ for the columns of $A$ (resp. $\left.H, U\right)$.

(1) [Initialize] Set $i=m, k=n$ and $U=\mathrm{I}_{n}$. If $m \leqslant n$ then set $l=1$ else set $l=m-n+1$.

(2) [Row finished?] If all the $a_{i j}$ with $j<k$ are zero, then if $a_{i k}<0$ replace column $A_{k}$ by $-A_{k}$, replace column $U_{k}$ by $-U_{k}$ and go to step 5 .

(3) [Choose non-zero entry] Pick among the non-zero $a_{i j}$, with $j \leqslant k$, one with the smallest absolute value, say $a_{i j_{0}}$. Then if $j_{0}<k$, exchange column $A_{k}$ with column $A_{j_{0}}$ and exchange column $U_{k}$ with column $U_{j_{0}}$. In addition, if $a_{i k}<0$ replace column $A_{k}$ by $-A_{k}$ and replace column $U_{k}$ by $-U_{k}$. Set $b=a_{i k}$.

(4) [Reduce] For $j=1, \ldots, k-1$ do the following: set $q=\left\lfloor a_{i k} / b\right\rceil$, and $A_{j}=A_{j}-q A_{k}$, $U_{j}=U_{j}-q U_{k}$. Then go to step 2 .

(5) [Final reductions] Set $b=a_{i k}$. If $b=0$, set $k=k+1$ and go to step 6. Otherwise, for $j>k$ do the following: set $q=\left\lfloor a_{i k} / b\right\rfloor$, and $A_{j}=A_{j}-q A_{k}, U_{j}=U_{j}-q U_{k}$.

(6) [Finished?] If $i=l$ then for $j=1, \ldots, n-k+1$ set $H_{j}=A_{j+k-1}$ and terminate the algorithm, else set $i=i-1, k=k-1$ and go to step 2 .

\section{Acknowledgments}

Special thanks to Frits Beukers for valuable hints that helped us with section 6. We also thank António Machiavelo, Ian Stewart and Arne Storjohann for helpful discussions.

\section{References}

[1] Cohen A M, Cuypers H and Sterk H (ed) 1999 Some Tapas of Computer Algebra (Algorithms and Computation in Mathematics vol 4) (Berlin: Springer)

[2] Cohen H 1993 A course in Computational Algebraic Number Theory (Graduate Texts in Mathematics vol 138) (Berlin: Springer)

[3] Cohen H 2000 Advanced Topics in Computational Number Theory (Graduate Texts in Mathematics vol 193) (New York: Springer)

[4] Dias A P S and Stewart I 2005 Linear equivalence and ODE-equivalence for coupled cell networks Nonlinearity 18 1003-20

[5] Field M 2004 Combinatorial dynamics Dyn. Syst. $19217-43$

[6] Golubitsky M and Stewart I 2006 Nonlinear dynamics of networks: the groupoid formalism Bull. Am. Math. Soc. 43 305-64

[7] Golubitsky M, Stewart I and Török A 2005 Patterns of synchrony in coupled cell networks with multiple arrows SIAM J. Appl. Dynam. Syst. 4 78-100

[8] Stewart I 2004 Network opportunity Nature 427 601-4

[9] Stewart I, Golubitsky M and Pivato M 2003 Symmetry groupoids and patterns of synchrony in coupled cell networks SIAM J. Appl. Dyn. Syst. 2 609-46

[10] Tutte W T 1984 Graph Theory (Encyclopedia of Mathematics and its Applications vol 21) (Menlo Park, CA: Addison-Wesley) 